\title{
ON THE NONLINEARITY OF QUANTUM DYNAMICAL ENTROPY
}

\author{
GEORGE ANDROULAKIS AND DUNCAN WRIGHT
}

\begin{abstract}
Linearity of a dynamical entropy means that the dynamical entropy of the $n$-fold composition of a dynamical map with itself is equal to $n$ times the dynamical entropy of the map for every positive integer $n$. We show that the quantum dynamical entropy introduced by Słomczyński and Życzkowski is nonlinear in the time interval between successive measurements of a quantum dynamical system. This is in contrast to Kolmogorov-Sinai dynamical entropy for classical dynamical systems, which is linear in time. We also compute the exact values of quantum dynamical entropy for the Hadamard walk with varying Lüders-von Neumann instruments and partitions.
\end{abstract}

\section{INTRODUCTION}

Entropy is a crucial concept in thermodynamics, dynamical systems and information theory. It was first introduced mathematically by Boltzmann near the end of the 19th century as a tool to measure disorder for the positions and velocities of gas molecules [4]. Almost eighty years later, Shannon became the father of the new field of information theory when he produced his groundbreaking works where he used entropy as a measure of information transfer between two sources [18, 19]. Dynamical entropy in classical systems can be seen from two distinct viewpoints: The information theoretic viewpoint (see e.g. [6]) which uses entropy rate of stochastic processes and the dynamical systems viewpoint (see e.g. [9]) which uses the Kolmogorov-Sinai (KS) dynamical entropy. We prove that the connection between entropy rate and KS entropy is seen through the symbolic dynamics of a stochastic process, which is a dynamical system with KS entropy equal to the entropy rate of the original stochastic process (see Subsection 2.3). On the other hand, entropy rate and KS entropy are inherently different as the former is probabilistic in nature and the latter is deterministic (see Subsection 2.4).

There have been many successful attempts to generalize KS entropy to a quantum dynamical entropy in [5, 2, 21, 1, 15] and more. The Connes-Narnhofer-Thirring (CNT) [5, Alicki-Lindblad-Fannes (ALF) 2], Accardi-Ohya-Watanabe (AOW) [1] and Kossakowski-Ohya-Watanabe (KOW) [15] entropies have had the most attention in the literature as they can be computed exactly for several examples of quantum dynamical systems. However we will investigate the Słomczyński-Życzkowski (SZ)

2000 Mathematics Subject Classification. Primary: 46L55, 94A17 ; Secondary: 37M25, 60G99, $82 \mathrm{C} 10$.

Key words and phrases. Quantum Dynamical Entropy; Quantum Random Walk.

This work is part of the second author's $\mathrm{PhD}$ thesis.

The second author was partially supported by a SPARC Graduate Research Grant from the Office of the Vice President for Research at the University of South Carolina. 
quantum dynamical entropy [21] which uses a semi-classical approach and was developed using the general notions of measurements, instruments, phase space and state space developed by Edwards [10] and Davies and Lewis [8, 7]. In contrast to both the CNT and ALF entropies, SZ dynamical entropy can obtain nonzero values for quantum systems with finite-dimensional Hilbert spaces. Quantum algorithms are a natural example where this property is desirable. To demonstrate the applicability of SZ dynamical entropy for quantum algorithms, we will introduce unitary quantum random walks which have been shown to be universal for quantum computation [16] and give exact computations of the SZ dynamical entropy for the Hadamard walk measured with Lüders-von Neumann instruments.

The paper is organized as follows: In Section 2 we recall the definitions of entropy rate and KS entropy. In Subsection 2.3 we introduce symbolic dynamics and establish the connection between the two notions of entropy and in Subsection 2.4 we show how they two differ. In Section 3 we recall the general notions of measurements through state space, phase space, observables and instruments. In Section 4 we recall the notions of SZ dynamical entropy and show that it is nonlinear in time intervals between successive measurements, in contrast to KS entropy for classical dynamical systems. In Section 5 we recall unitary quantum random walks and, in particular, the Hadamard walk. Lastly, in Section [6 we give exact calculations of the SZ dynamical entropy of the Hadamard walk with varying instruments, partitions and time intervals between successive measurements. These calculations verify the nonlinearity of the SZ dynamical entropy via an explicit example (see Theorem 6.3).

\section{Entropy in Classical Systems}

2.1. Entropy from the Dynamical Systems Point of View. Let $(\Omega, \Sigma)$ be a measurable space. Define $\mathcal{P}_{a r}(\Omega)$ to be the collection of all finite or countably infinite measurable partitions of $\Omega$. Define a partial ordering on $\mathcal{P}_{a r}(\Omega)$ such that, for any $\mathcal{C}, \mathcal{D} \in \mathcal{P}_{a r}(\Omega), \mathcal{D} \leq \mathcal{C}$ whenever, for every $D \in \mathcal{D}$ there exists $\mathcal{C}_{D} \subseteq \mathcal{C}$ such that $D=\cup \mathcal{C}_{D}$. If $\mathcal{D} \leq \mathcal{C}$ we say that $\mathcal{C}$ is finer than $\mathcal{D}$ or that $\mathcal{D}$ is coarser than $\mathcal{C}$.

Whenever $\Omega$ is finite or countably infinite and $\Sigma=\mathcal{P}(\Omega)$, where $\mathcal{P}(\Omega)$ is the power set of $\Omega$, we call $(\Omega, \Sigma)$ (or simply $\Omega$ ) a discrete space. In that case, we will refer to the partition of $\Omega$ into singletons $\{\{\omega\}\}_{\omega \in \Omega}$ as the atomic partition. Whenever $\Omega$ is a discrete space it is clear that the atomic partition $\mathcal{A}$ of $\Omega$ is countable and measurable; i.e. $\mathcal{A} \in \mathcal{P}_{\text {ar }}(\Omega)$. Furthermore, in this case, $\mathcal{A}$ is the finest partition in $\mathcal{P}_{a r}(\Omega)$; i.e. $\mathcal{C} \leq \mathcal{A}$ for any $\mathcal{C} \in \mathcal{P}_{a r}(\Omega)$.

For any $\mathcal{C}, \mathcal{D} \in \mathcal{P}_{a r}(\Omega)$, the join (or least upper bound) of $\mathcal{C}$ and $\mathcal{D}$ is given by the partition $\mathcal{C} \vee \mathcal{D}$ which contains all sets of the form $C \cap D$ for all $C \in \mathcal{C}$ and $D \in \mathcal{D}$. Given a finite collection of partitions $\left\{\mathcal{C}_{k}\right\}_{k=1}^{n} \subseteq \mathcal{P}_{a r}(\Omega)$, we define the join $\vee_{k=1}^{n} \mathcal{C}_{k}$ to be the partition containing exactly the sets of the form $\cap_{k=1}^{n} C_{k}$, where $C_{k} \in \mathcal{C}_{k}$ for all $1 \leq k \leq n$.

Fix a probability space $(\Omega, \Sigma, \mu)$. Given any partition $\mathcal{C} \in \mathcal{P}_{a r}(\Omega)$ we define the entropy of $\mathcal{C}$ by

$$
H_{\mu}(\mathcal{C}):=\sum_{C \in \mathcal{C}} \eta(\mu(C))
$$


where $\eta:[0, \infty) \rightarrow[0, \infty)$ is given by $\eta(x)=-x \ln x$, for $x>0$ and we agree that $\eta(0)=0$. When there is no confusion about the probability measure in question, we will simply write $H(\mathcal{C})$ instead of $H_{\mu}(\mathcal{C})$.

Remark 2.1 ([9, Page 23]). It is well known that $\eta$ is countably subadditive; i.e. $\eta\left(\sum_{n} a_{n}\right) \leq \sum_{n} \eta\left(a_{n}\right)$ for any nonnegative sequence $\left\{a_{n}\right\}_{n}$. This gives that for any probability space $(\Omega, \Sigma, \mu)$ and any two partitions $\mathcal{C}, \mathcal{D} \in \mathcal{P}_{\text {ar }}(\Omega)$ satisfying $\mathcal{D} \leq \mathcal{C}$, we have that $H(\mathcal{D}) \leq H(\mathcal{C})$.

Fix a probability space $(\Omega, \Sigma, \mu)$. Recall that, for any two sets $C, D \in \Sigma$, the conditional probability of $\boldsymbol{C}$ given $\boldsymbol{D}$ is given by $\mu(C \mid D):=\mu(C \cap D) / \mu(D)$. Given two partitions, $\mathcal{C}, \mathcal{D} \in \mathcal{P}_{a r}(\Omega)$, the conditional entropy of $\mathcal{C}$ given $\mathcal{D}$ is given by

$$
H(\mathcal{C} \mid \mathcal{D}):=\sum_{D \in \mathcal{D}} \mu(D) \sum_{C \in \mathcal{C}} \eta(\mu(C \mid D))=-\sum_{\substack{C \in \mathcal{C} \\ D \in \mathcal{D}}} \mu(C \cap D) \ln (\mu(C \mid D)) .
$$

The so-called chain rule follows.

Theorem 2.2 (Chain Rule, [9, Equation 1.4.3]). Let $(\Omega, \Sigma, \mu)$ be a probability space and $\mathcal{C}, \mathcal{D} \in \mathcal{P}_{\text {ar }}(\Omega)$. Then

$$
H(\mathcal{C} \vee \mathcal{D})=H(\mathcal{D})+H(\mathcal{C} \mid \mathcal{D})
$$

More generally, given a finite collection of partitions $\mathcal{C}_{0}, \ldots, \mathcal{C}_{n} \in \mathcal{P}_{\text {ar }}(\Omega)$, we have

$$
H\left(\vee_{k=0}^{n} \mathcal{C}_{k}\right)=H\left(\mathcal{C}_{0}\right)+\sum_{k=1}^{n} H\left(\mathcal{C}_{k} \mid \vee_{\ell=0}^{k-1} \mathcal{C}_{\ell}\right)
$$

Also, from the definition of conditional entropy (Equation (1)) and the countable subadditivity of $\eta$ (Remark 2.1) we have, for any $\mathcal{B}, \mathcal{C}, \mathcal{D} \in \mathcal{P}_{a r}(\Omega)$ satisfying $\mathcal{B} \leq \mathcal{D}$, that

$$
0 \leq H(\mathcal{C} \mid \mathcal{D}) \leq H(\mathcal{C} \mid \mathcal{B})
$$

See [9, Section 1.4] for more details on conditional entropy of partitions. The following theorem will be used throughout the article. In the proof we will use the well known Césaro mean Theorem which states that, for any sequence of real numbers $\left\{a_{n}\right\}_{n=0}^{\infty}$ converging to some element, $a \in \mathbb{R} \cup\{\infty\}$, the sequence $\left\{b_{n}\right\}_{n=0}^{\infty}$ given by $b_{n}=$ $\frac{1}{n} \sum_{k=0}^{n-1} a_{k}$, for each $n \in \mathbb{N}$, also converges to $a$.

Theorem 2.3. Let $(\Omega, \Sigma, \mu)$ be a probability space and $\left\{\mathcal{C}_{n}\right\}_{n=0}^{\infty} \subseteq \mathcal{P}_{a r}(\Omega)$ be a sequence of partitions. If $\lim _{n \rightarrow \infty} H\left(\mathcal{C}_{n} \mid \vee_{k=0}^{n-1} \mathcal{C}_{k}\right)=a$, then $\lim _{n \rightarrow \infty} \frac{1}{n} H\left(\vee_{k=0}^{n-1} \mathcal{C}_{k}\right)=a$.

Proof. Set $a_{0}=H\left(\mathcal{C}_{0}\right)$ and $a_{n}=H\left(\mathcal{C}_{n} \mid \vee_{k=0}^{n-1} \mathcal{C}_{k}\right)$, for each $n \in \mathbb{N}$. Then, by assumption, $a_{n}$ converges to $a \in \mathbb{R} \cup\{\infty\}$. For each $n \in \mathbb{N}$, set $b_{n}=\frac{1}{n} \sum_{k=0}^{n-1} a_{k}$. Then, by Theorem 2.2, $b_{n}=\frac{1}{n} H\left(\vee_{k=0}^{n-1} \mathcal{C}_{k}\right)$ which converges to $a$ by the Césaro mean Theorem.

Next we wish to define the Kolmogorov-Sinai (KS) dynamical entropy. In order to do so, we must first introduce some dynamics on the probability space $(\Omega, \Sigma, \mu)$. This role will be played by a measurable map $f: \Omega \rightarrow \Omega$. We call the quadruple $(\Omega, \Sigma, \mu, f)$ a dynamical system (DS). Furthermore, whenever $\mu(A)=\mu\left(f^{-1}(A)\right)$ for all $A \in \Sigma$, we say that $\boldsymbol{\mu}$ is $\boldsymbol{f}$-invariant and call the DS $(\Omega, \Sigma, \mu, f)$ stationary. 
Fix a $\operatorname{DS}(\Omega, \Sigma, \mu, f)$ and a partition $\mathcal{C} \in \mathcal{P}_{\text {ar }}(\Omega)$. For each $k \in \mathbb{N}_{0}$, define the $k^{\text {th }}$-preimage of $\mathcal{C}$ under $f$ by $f^{-k}(\mathcal{C}):=\left\{f^{-k}(C)\right\}_{C \in \mathcal{C}}$, where $f^{0}$ denotes the identity map. Note that, for each $\mathcal{C} \in \mathcal{P}_{a r}(\Omega), f^{-1}(\mathcal{C}) \in \mathcal{P}_{a r}(\Omega)$ and hence $f^{-k}(\mathcal{C}) \in \mathcal{P}_{a r}(\Omega)$ for every $k \in \mathbb{N}_{0}$. The Kolmogorov-Sinai $(\mathrm{KS})$ entropy of $(\Omega, \Sigma, \boldsymbol{\mu}, \boldsymbol{f})$ with respect to $\mathcal{C}$ is given by

$$
h^{K S}(f, \mathcal{C})=\lim _{n \rightarrow \infty} \frac{1}{n} H\left(\vee_{k=0}^{n-1} f^{-k}(\mathcal{C})\right)
$$

whenever this limit exists.

Remark 2.4. Given a $D S(\Omega, \Sigma, \mu, f)$ and a partition $\mathcal{C} \in \mathcal{P}_{\text {ar }}(\Omega)$, it is clear that $\vee_{k=0}^{n-1} f^{-k}(\mathcal{C})$ consists exactly of sets of the form $f^{-(n-1)}\left(A_{n-1}\right) \cap \cdots \cap f^{-1}\left(A_{1}\right) \cap A_{0}$ for all $A_{0}, \ldots, A_{n-1} \in \mathcal{C}$.

From Theorem 2.3, we have

$$
h^{K S}(f, \mathcal{C})=\lim _{n \rightarrow \infty} H\left(f^{-n}(\mathcal{C}) \mid \vee_{k=0}^{n-1} f^{-k}(\mathcal{C})\right),
$$

whenever this limit exists.

Corollary 2.5. Let $(\Omega, \Sigma, \mu, f)$ be a stationary $D S$ and $\mathcal{C} \in \mathcal{P}_{a r}(\Omega)$. Then the limit in Equation (44), and hence the limit in Equation (3), exists and $h^{K S}(f, \mathcal{C})=$ $\lim _{n \rightarrow \infty} H\left(f^{-n}(\mathcal{C}) \mid \vee_{k=0}^{n-1} f^{-k}(\mathcal{C})\right)$.

Proof. For each $n \in \mathbb{N}$ with $n \geq 2$, we have

$$
\begin{aligned}
H\left(f^{-n}(\mathcal{C}) \mid \vee_{k=0}^{n-1} f^{-k}(\mathcal{C})\right) & \leq H\left(f^{-n}(\mathcal{C}) \mid \vee_{k=1}^{n-1} f^{-k}(\mathcal{C})\right) \quad \text { by (2) } \\
& =H\left(f^{-(n-1)}(\mathcal{C}) \mid \vee_{k=0}^{n-2} f^{-k}(\mathcal{C})\right) \quad \text { since }(\Omega, \Sigma, \mu, f) \text { is stationary. }
\end{aligned}
$$

Therefore $H\left(f^{-n}(\mathcal{C}) \mid \vee_{k=0}^{n-1} f^{-k}(\mathcal{C})\right)$ is a decreasing sequence which is bounded below by zero and hence converges. By Theorem 2.3 ,

$$
h^{K S}(f, \mathcal{C})=\lim _{n \rightarrow \infty} H\left(f^{-n}(\mathcal{C}) \mid \vee_{k=0}^{n-1} f^{-k}(\mathcal{C})\right) .
$$

Remark 2.6. In the literature it is common to only refer to $(\Omega, \Sigma, \mu, f)$ as a $D S$ whenever $\mu$ is $f$-invariant. Although this convention has its benefits, as evidenced by Corollary [2.5, we find it restrictive and do not adopt it here.

Finally, the $\mathrm{KS}$ entropy of $(\Omega, \Sigma, \mu, f)$ is given by

$$
h^{K S}(f)=\sup _{\substack{\mathcal{C} \in \mathcal{P} \text { ar }(\Omega) \\ H(\mathcal{C})<\infty}} h^{K S}(f, \mathcal{C}) .
$$

Remark 2.7. Fix a dynamical system $(\Omega, \Sigma, \mu, f)$. In many instances, KS entropy is taken as the sup over only finite partitions. However, the two definitions are equivalent (see [9, Page 102]). Furthermore, it is remarked in [9, Page 61] that the restriction of the sup in Equation (5) to include only those partitions, $\mathcal{C}$, satisfying $H(\mathcal{C})<\infty$ is natural because otherwise it is possible to obtain infinite $K S$ entropy for the identity transformation. This is due to the fact that $H(f, \mathcal{C})=\infty$ whenever $H(\mathcal{C})=\infty$. 
For a more detailed exposition on dynamical entropy and classical dynamical systems (with invariant measures), we refer the reader to the book of Walters [22]. For extensions of the results of Walters to include infinite partitions with finite entropy, the reader is referred to the book of Downarowicz [9].

2.2. Entropy from the Information Theoretic Point of View. Next we look at entropy of random variables. Let $(\Omega, \Sigma, \mu)$ be a probability space and let $(E, \mathcal{E})$ be a measurable space. An $(\Omega, \boldsymbol{E})$ random variable $X$ is a measurable map $X: \Omega \rightarrow E$. For any $S \in \mathcal{E}$, the probability that $X$ takes values in $S$ is given by $\mu(X \in S):=$ $\mu\left(X^{-1}(S)\right)$. We say that the random variable $\boldsymbol{X}$ is discrete whenever its range $E$ is a discrete space. In that case, $X$ is determined by its probability mass function $(\mathrm{pmf}) p_{X}: E \rightarrow[0,1]$ given by $p_{X}(x)=\mu(X=x)$ for each $x \in E$. We will simply write $p(x)$ as opposed to $p_{X}(x)$ when there is no confusion about the random variable. Given a discrete random variable $X$, the collection

$$
\mathcal{C}_{X}:=\left\{X^{-1}(\{x\})\right\}_{x \in E}
$$

is a countable measurable partition of $\Omega$; i.e. $\mathcal{C}_{X} \in \mathcal{P}_{a r}(\Omega)$. The entropy of a discrete random variable $X$ is given by the entropy of $\mathcal{C}_{X}$ and is related to its pmf by the equation

$$
H_{\mu}(X):=H_{\mu}\left(\mathcal{C}_{X}\right)=\sum_{x \in E} \eta(p(x))
$$

When there is no confusion about the probability measure in question, we will simply write $H(X)$ instead of $H_{\mu}(X)$.

Given a finite collection, $\left(X_{k}\right)_{k=0}^{n}$, of $(\Omega, E)$ discrete random variables the joint pmf of $\left(\boldsymbol{X}_{\mathbf{0}}, \ldots, \boldsymbol{X}_{\boldsymbol{n}}\right)$ is given by $p_{X_{0}, \ldots, X_{n}}\left(x_{0}, \ldots, x_{n}\right)=\mu\left(X_{0}=x_{0}, \ldots, X_{n}=x_{n}\right)$ for all $x_{0}, \ldots, x_{n} \in E$. Furthermore, $\left(X_{0}, \ldots, X_{n}\right)$ is a discrete $\left(\Omega, E^{n+1}\right)$ random variable and $\mathcal{C}_{\left(X_{0}, \ldots, X_{n}\right)}=\vee_{k=0}^{n} \mathcal{C}_{X_{k}}$. Therefore the entropy of $\left(X_{0}, \ldots, X_{n}\right)$ is given by Equation (6) and is related to its joint pmf by the equation

$$
H\left(X_{0}, \ldots, X_{n}\right):=H\left(\vee_{k=0}^{n} \mathcal{C}_{X_{k}}\right)=\sum_{\substack{x_{k} \in E \\ 0 \leq k \leq n}} \eta\left(p_{X_{0}, \ldots, X_{n}}\left(x_{0}, \ldots, x_{n}\right)\right) .
$$

The conditional pmf of $\boldsymbol{X}_{\boldsymbol{n}}$ given $\left(\boldsymbol{X}_{\mathbf{0}}, \ldots, \boldsymbol{X}_{\boldsymbol{n}-1}\right)$ is given by

$$
\begin{aligned}
p_{X_{n} \mid\left(X_{0}, \ldots, X_{n-1}\right)}\left(x_{n} \mid x_{0}, \ldots, x_{n-1}\right): & =\mu\left(X_{n}=x_{n} \mid X_{0}=x_{0}, \ldots, X_{n-1}=x_{n-1}\right) \\
& =\frac{p_{X_{0}, \ldots, X_{n}}\left(x_{0}, \ldots, x_{n}\right)}{p_{X_{0}, \ldots, X_{n-1}}\left(x_{0}, \ldots, x_{n-1}\right)} \quad \text { for all } x_{0}, \ldots, x_{n} \in E .
\end{aligned}
$$

Whenever there is no confusion about the random variables in question we simply write $p\left(x_{0}, \ldots, x_{n}\right)$ for the joint pmf and $p\left(x_{n} \mid x_{0}, \ldots, x_{n-1}\right)$ for the conditional pmf. The conditional entropy of $\boldsymbol{X}_{\boldsymbol{n}}$ given $\left(\boldsymbol{X}_{\mathbf{0}}, \ldots, \boldsymbol{X}_{n-1}\right)$ is given by the conditional entropy of $C_{X_{n}}$ given $\vee_{k=0}^{n-1} C_{X_{k}}$ and is related to their conditional pmf by the equation

$$
\begin{aligned}
H\left(X_{n} \mid X_{0}, \ldots, X_{n-1}\right): & =H\left(\mathcal{C}_{X_{n}} \mid \vee_{k=0}^{n-1} \mathcal{C}_{X_{k}}\right) \\
& =\sum_{\substack{x_{k} \in E \\
0 \leq k \leq n-1}} p\left(x_{0}, \ldots, x_{n-1}\right) \sum_{x_{n} \in E} \eta\left(p\left(x_{n} \mid x_{0}, \ldots, x_{n-1}\right)\right) .
\end{aligned}
$$

See [6, Section 2.2] for more details on conditional entropy of random variables. 
Remark 2.8. The entropy and conditional entropy of non-discrete random variables can be defined similarly to Equations (6) and (8), respectively, by using integration and probability distribution functions instead of sums and pmfs. However, we are mainly interested in discrete random variables here.

Next we turn to entropy rate of stochastic processes. If $(\Omega, \Sigma, \mu)$ is a probability space and $(E, \mathcal{E})$ is a measurable space, then a (discrete time) $(\Omega, \boldsymbol{E})$ stochastic process is an indexed sequence of $(\Omega, E)$ random variables. Throughout this paper we will only consider discrete time stochastic processes and the sequences will all be indexed by $\mathbb{N}_{0}:=\mathbb{N} \cup\{0\}$, where the index is meant to represent time. Whenever $(E, \mathcal{E})$ is a discrete space the stochastic process, $\mathbf{X}=\left(X_{n}\right)_{n=0}^{\infty}$, is determined by its joint pmf, denoted by $p_{\mathbf{X}}$, and given by $p_{\mathbf{X}}\left(x_{0}, \ldots, x_{n}\right)=p_{X_{0}, \ldots, X_{n}}\left(x_{0}, \ldots, x_{n}\right)$ for each $n \in \mathbb{N}_{0}$ and $x_{0}, \ldots, x_{n} \in E$. Given a stochastic process of discrete random variables, the entropy of a finite initial subsequence is given by Equation (17) and the conditional entropy of the $n^{\text {th }}$ term given all the previous ones is given by Equation (8).

Fix a discrete $(\Omega, E)$ stochastic process $\mathbf{X}$. Then, from the classical random walk perspective, we interpret $p_{X_{0}}(x)$ as the probability that a random walker inhabits the site $x$ initially at time 0 and $p_{X_{n}}(x)$ as the probability that a random walker inhabits the site $x$ at time $n$, for any $x \in E$ and $n \in \mathbb{N}$. Furthermore, for any $x_{0}, \ldots, x_{n} \in E$ and $n \in \mathbb{N}$, we interpret $\left.p_{(} x_{0}, \ldots, x_{n}\right)$ as the probability that a random walker takes the path $x_{0} \rightarrow x_{1} \rightarrow \cdots \rightarrow x_{n}$ at times $0,1, \ldots, n$.

A stochastic process $\left(X_{n}\right)_{n=0}^{\infty}$ of discrete random variables is called stationary whenever its joint pmf is invariant with respect to shifts of the time index; i.e.

$$
\mu\left(X_{0}=x_{0}, \ldots, X_{n}=x_{n}\right)=\mu\left(X_{l}=x_{0}, \ldots, X_{n+l}=x_{n}\right),
$$

for all $n, l \in \mathbb{N}_{0}$ and $x_{0}, \ldots, x_{n} \in E$. In the literature, a stationary stochastic process is sometimes referred to as being time invariant (see e.g. [6, Page 61]).

A simple example of a stochastic process is one in which each random variable depends only on the one proceeding it in the sequence; i.e.

$$
\mu\left(X_{n+1}=x_{n+1} \mid X_{0}=x_{0}, \ldots, X_{n}=x_{n}\right)=\mu\left(X_{n+1}=x_{n+1} \mid X_{n}=x_{n}\right),
$$

for all $n \in \mathbb{N}_{0}$ and $x_{0}, \ldots, x_{n+1} \in E$. A stochastic process of discrete random variables which satisfies Equation (9) is referred to as a Markov process. In particular, we are interested in those discrete Markov processes, $\mathbf{X}=\left(X_{n}\right)_{n=0}^{\infty}$, whose conditional pmfs do not vary with time; i.e.

$$
\mu\left(X_{1}=x \mid X_{0}=y\right)=\mu\left(X_{n+1}=x \mid X_{n}=y\right) \text { for all } x, y \in E \text {, and } n \in \mathbb{N}_{0} .
$$

In this case, we will set $p_{x, y}:=\mu\left(X_{1}=x \mid X_{0}=y\right)$ and define the $|E| \times|E|$ matrix $P$ to have $(x, y)$-entry given by $p_{x, y}$, for all $x, y \in E$. Then $P$ is a transition (column-stochastic) matrix; i.e. for all $x, y \in E, 0 \leq p_{x, y} \leq 1$ and, for all $y \in E$, $\sum_{x \in E} p_{x, y}=1$. From the classical random walk perspective, the $(x, y)$-entry of $P$, $p_{x, y}$, is interpreted as the conditional probability that a random walker will move in one step from site $y$ to site $x$.

The entropy rate of a stochastic process $\mathrm{X}=\left(\boldsymbol{X}_{n}\right)_{n=0}^{\infty}$ is given by

$$
H(\mathbf{X}):=\lim _{n \rightarrow \infty} \frac{1}{n} H\left(X_{0}, \ldots, X_{n-1}\right),
$$

whenever this limit exists. 
Another quantity, which is often equal to the entropy rate, is given by

$$
H^{\prime}(\mathbf{X}):=\lim _{n \rightarrow \infty} H\left(X_{n} \mid X_{0}, \ldots, X_{n-1}\right),
$$

whenever this limit exists. The two quantities $H(\mathbf{X})$ and $H^{\prime}(\mathbf{X})$ correspond to two different interpretations of entropy rate. The first is interpreted as the average entropy of the first $n$ random variables and the second as the entropy of the last random variable given the past. The following result shows the relationship between $H(\mathbf{X})$ and $H^{\prime}(\mathbf{X})$.

Corollary 2.9. Let $\mathbf{X}=\left(X_{n}\right)_{n=0}^{\infty}$ be a stochastic process. If the limit in Equation (12) exists, then the limit in Equation (11) also exists and $H(\mathbf{X})=H^{\prime}(\mathbf{X})$.

Proof. By the definitions of $H\left(X_{0}, \ldots, X_{n}\right)$ and $H\left(X_{n} \mid X_{0}, \ldots, X_{n-1}\right)$ in Equations (7) and (8), respectively, this is simply a restatement of Theorem 2.3 .

The following is another corollary for stationary stochastic processes which is also proved in [6, Theorem 4.2.2].

Corollary 2.10. Let $\mathbf{X}$ be a stationary stochastic process. Then the limits in Equations (11) and (12) both exist and $H(\mathbf{X})=H^{\prime}(\mathbf{X})$.

Proof. The proof is similar to the proof of Corollary 2.5. For each $n \in \mathbb{N}$ with $n \geq 2$, we have

$$
\begin{aligned}
H\left(X_{n} \mid X_{0}, \ldots, X_{n-1}\right) & \leq H\left(X_{n} \mid X_{1}, \ldots, X_{n-1}\right) \quad \text { by (2) } \\
& =H\left(X_{n-1} \mid X_{0}, \ldots, X_{n-2}\right) \quad \text { since } \mathbf{X} \text { is stationary. }
\end{aligned}
$$

Therefore $H\left(X_{n} \mid X_{0}, \ldots, X_{n-1}\right)$ is a decreasing sequence which is bounded below by zero and hence converges. By Equations (7) and (8), and Theorem 2.3, $H(\mathbf{X})=$ $H^{\prime}(\mathbf{X})$.

Next we look at the entropy rate of discrete Markov processes governed by a transition matrix; i.e. the discrete Markov processes satisfying Equation (10). In this case, given a discrete measurable space $(E, \mathcal{P}(E))$, we will represent a probability measure $\mu$ on $(E, \mathcal{P}(E))$ as the column vector $\mu=\left\{\mu_{e}\right\}_{e \in E}$, where $\mu_{e}:=\mu(\{e\})$ for each $e \in E$, which we will refer as a probability vector. Then, given a transition matrix $P$ on $E$, we define $P \mu$ by matrix multiplication. We say that $\boldsymbol{\mu}$ is $\boldsymbol{P}$-invariant whenever $P \mu=\mu$. In particular, whenever $\mathbf{X}$ is an $(\Omega, E)$ Markov process governed by the transition matrix $P$, we take the initial measure $\mu$ to be $p_{X_{0}}$. In this case, notice that $\mathbf{X}$ is stationary if and only if $p_{X_{0}}$ is $P$-invariant. The following theorem gives a simplification of the entropy rate for Markov processes governed by a transition matrix.

Theorem 2.11. Let $\mathbf{X}$ be a discrete $(\Omega, E)$ Markov process governed by the transition matrix $P$ and set $\mu=p_{X_{0}}$. Then

$$
H(\mathbf{X})=\lim _{n \rightarrow \infty} \sum_{y \in E}\left(P^{n} \mu\right)_{y} \sum_{x \in E} \eta\left(p_{x, y}\right)
$$

whenever the limit exists. Moreover, if $\mathbf{X}$ is stationary, then

$$
H(\mathbf{X})=\sum_{y \in E} \mu_{y} \sum_{x \in E} \eta\left(p_{x, y}\right) .
$$


Proof. Since $\mathbf{X}$ is a Markov process governed by the transition matrix $P$ we have

$$
H\left(X_{n+1} \mid X_{0}, \ldots, X_{n}\right)=H\left(X_{n+1} \mid X_{n}\right)=\sum_{y \in E} p_{X_{n}}(y) \sum_{x \in E} \eta\left(p_{x, y}\right) \quad \text { for each } n \in \mathbb{N},
$$

where the second equality follows from Equation (8) . Then from the definition of matrix multiplication, for each $e_{n} \in E$, we have

$$
p_{X_{n}}\left(e_{n}\right)=\sum_{\substack{e_{k} \in E \\ 0 \leq k \leq n-1}} p_{X_{0}}\left(e_{0}\right) \prod_{k=1}^{n} p_{e_{k}, e_{k-1}}=\left(P^{n} \mu\right)_{e_{n}} .
$$

Therefore

$$
H(\mathbf{X})=\lim _{n \rightarrow \infty} \sum_{y \in E}\left(P^{n} \mu\right)_{y} \sum_{x \in E} \eta\left(p_{x, y}\right)
$$

whenever the limit exists. The moreover statement is immediate because $P^{n} \mu=\mu$ for all $n \in \mathbb{N}_{0}$ whenever $\mathbf{X}$ is stationary.

Remark 2.12. Certain cases of Theorem 2.11 appear frequently in the literature, but to the best of our knowledge we have not seen it presented in the generality of above. For instance, it can be seen for the case where $\mu$ is P-invariant in [6, Theorem 4.2.4] or [22, Theorem 4.26].

In the literature, given a transition matrix $P$ on a discrete measurable space $(E, \mathcal{P}(E))$ with a unique invariant probability vector $\mu$, it is common to set

$$
H(P):=\sum_{y \in E} \mu_{y} \sum_{x \in E} \eta\left(p_{x, y}\right),
$$

and refer to $H(P)$ as the entropy of $\boldsymbol{P}$. As it is shown in Theorem 2.11, the entropy, $H(P)$, of $P$ is equal to the entropy rate, $H(\mathbf{X})$, of any stationary Markov process, $\mathbf{X}$, governed by the transition matrix $P$ such that $p_{X_{0}}=\mu$.

2.3. The connection between entropy rate and $\mathbf{K S}$ entropy. Let $(\Omega, \Sigma, \mu)$ be a probability space, $(E, \mathcal{E})$ a measurable space and $\mathbf{X}=\left(X_{n}\right)_{n=0}^{\infty}$ an $(\Omega, E)$ stochastic process. Consider the measurable space $\left(E^{*}, \mathcal{E}^{*}\right)$, where $E^{*}:=E^{\mathbb{N}_{0}}$ and $\mathcal{E}^{*}:=\sigma\left(\cup_{n=0}^{\infty} \mathcal{E}^{n+1}\right)$. For all $n \in \mathbb{N}_{0}$, collection of integer times $0 \leq t_{0}<t_{1}<\cdots<t_{n}$ and $A_{0}, \ldots, A_{n} \in \mathcal{E}$, we define the cylinder set

$$
C\left(\begin{array}{ccc}
A_{0} & \cdots & A_{n} \\
t_{0} & \cdots & t_{n}
\end{array}\right):=\left\{x=\left(x_{i}\right)_{i \in \mathbb{N}_{0}} \in E^{*}: x_{t_{k}} \in A_{k} \text { for } k \in\{0, \ldots, n\}\right\} .
$$

For $\mathcal{C} \in \mathcal{P}_{a r}(E)$, we say $C\left(\begin{array}{ccc}A_{0} & \cdots & A_{n} \\ t_{0} & \cdots & t_{n}\end{array}\right)$ is a $\mathcal{C}$-cylinder set if $A_{0}, \ldots, A_{n} \in \mathcal{C}$. Also, we define the partition, $\widehat{\mathcal{C}} \in \mathcal{P}_{a r}\left(E^{*}\right)$, by

$$
\widehat{\mathcal{C}}:=\left\{C\left(\begin{array}{c}
A \\
0
\end{array}\right)\right\}_{A \in \mathcal{C}}
$$

and the set

$$
\widehat{\mathcal{P}_{a r}(E)}:=\left\{\widehat{\mathcal{C}}: \mathcal{C} \in \mathcal{P}_{a r}(E)\right\} \subset \mathcal{P}_{a r}\left(E^{*}\right)
$$

Notice that the collection of all cylinder sets in $E^{*}$ is a $\pi$-system which generates the $\sigma$-algebra $\mathcal{E}^{*}$. Therefore, any measure on $\left(E^{*}, \mathcal{E}^{*}\right)$ is uniquely defined by its values on the cylinder sets. We define the process-dependent measure, $\mu^{\mathbf{X}}$, on the cylinder sets by

$$
\mu^{\mathbf{X}}\left(C\left(\begin{array}{ccc}
A_{0} & \cdots & A_{n} \\
t_{0} & \cdots & t_{n}
\end{array}\right)\right)=\mu\left(\cap_{k=0}^{n}\left(X_{t_{k}} \in A_{k}\right)\right)
$$


for all $n \in \mathbb{N}_{0}, 0 \leq t_{0}<\cdots<t_{n}$, and $A_{0}, \ldots, A_{n} \in \mathcal{E}$. We interpret $\mu^{\mathbf{X}}\left(C\left(\begin{array}{ccc}A_{0} & \cdots & A_{n} \\ t_{0} & \cdots & t_{n}\end{array}\right)\right)$ as the probability that a random walker, governed by the stochastic process $\mathbf{X}$, is in the set $A_{0}$ at time $t_{0}$ and takes the path $A_{0} \rightarrow A_{1} \rightarrow \cdots \rightarrow A_{n}$ at times $t_{0}, \ldots, t_{n}$.

Remark 2.13. Fix an $(\Omega, E)$ stochastic process $\mathbf{X}, A_{0}, \ldots, A_{n} \in \mathcal{E}$, integer times $0 \leq t_{0}<\cdots<t_{n}$ and $t \in \mathbb{N} \backslash\left\{t_{0}, \ldots, t_{n}\right\}$ such that $t_{i}<t<t_{i+1}$ for some $0 \leq i<n$. Then, since $\mu\left(\cap_{k=0}^{n}\left(X_{t_{k}} \in A_{k}\right)\right)=\mu\left(\cap_{k=0}^{n}\left(X_{t_{k}} \in A_{k}\right) \cap\left(X_{t} \in E\right)\right)$, we have that

$$
\mu^{\mathbf{X}}\left(C\left(\begin{array}{ccc}
A_{0} & \cdots & A_{n} \\
t_{0} & \cdots & t_{n}
\end{array}\right)\right)=\mu^{\mathbf{X}}\left(C\left(\begin{array}{ccccccc}
A_{0} & \cdots & A_{i} & E & A_{i+1} & \cdots & A_{n} \\
t_{0} & \cdots & t_{i} & t & t_{i+1} & \cdots & t_{n}
\end{array}\right)\right) .
$$

Similarly, if $t<t_{0}$ or $t>t_{n}$. Therefore the measure $\mu^{\mathbf{x}}$ is well defined.

We define the shift map $s: E^{*} \rightarrow E^{*}$ by $s(x)=y$ where $y_{i}=x_{i+1}$, for each $i \in \mathbb{N}_{0}$, and refer to the quadruple, $\left(E^{*}, \mathcal{E}^{*}, \mu^{\mathbf{X}}, s\right)$, as the symbolic dynamics of $\mathbf{X}$. Notice that $\left(E^{*}, \mathcal{E}^{*}, \mu^{\mathbf{X}}, s\right)$ is a DS and thus its partition dependent and independent $\mathrm{KS}$ entropies are given by Equations (3) and (5), respectively.

Of particular interest is the $\mathrm{KS}$ entropy of $\left(E^{*}, \mathcal{E}^{*}, \mu^{\mathbf{X}}, s\right)$ with respect to the partitions in $\widehat{\mathcal{P}_{a r}(E)}$. For each $\mathcal{C} \in \mathcal{P}_{a r}(E)$, define the $(\Omega, \mathcal{C})$ stochastic process $\mathbf{X}_{\mathcal{C}}=\left(X_{n}^{\mathcal{C}}\right)_{n=0}^{\infty}$ where, for each $n \in \mathbb{N}_{0}$ and $\omega \in \Omega, X_{n}^{\mathcal{C}}(\omega)=A$ whenever $X_{n}(\omega) \in A$; i.e. $X_{n}^{\mathcal{C}}=i_{\mathcal{C}} \circ X_{n}$, where $i_{\mathcal{C}}: E \rightarrow \mathcal{C}$ is the natural map that assigns to each $e \in E$ the unique $A \in \mathcal{C}$ such that $e \in A$. Since the values of $\mathbf{X}_{\mathcal{A}}$ are singletons, it is clear that $\mathbf{X}$ can be identified with $\mathbf{X}_{\mathcal{A}}$ whenever $\mathcal{A}$ is the atomic partition of the discrete space $E$. The following proposition shows that the KS entropy of $\left(E^{*}, \Sigma^{*}, \mu^{\mathbf{X}}, s\right)$ with respect to $\widehat{\mathcal{C}}$ and the entropy rate of $\mathbf{X}_{\mathcal{C}}$ are equal.

Proposition 2.14. Let $(\Omega, \Sigma, \mu)$ be a probability space, $(E, \mathcal{E})$ a measurable space, $\mathbf{X}$ an $(\Omega, E)$ stochastic process and $\left(E^{*}, \Sigma^{*}, \mu^{\mathbf{X}}, s\right)$ the symbolic dynamics of $\mathbf{X}$. Then for each $\mathcal{C} \in \mathcal{P}_{\text {ar }}(E), H\left(\mathbf{X}_{\mathcal{C}}\right)=h^{K S}(s, \widehat{\mathcal{C}})$. In particular, whenever $E$ is a discrete space, $H(\mathbf{X})=h^{K S}(s, \widehat{\mathcal{A}})$, where $\mathcal{A}$ is the atomic partition of $E$.

Proof. Notice that, for each $n \in \mathbb{N}_{0}$, the collection of all $\mathcal{C}$-cylinder sets of initial length $n+1$ is given by $\vee_{k=0}^{n} s^{-k}(\widehat{\mathcal{C}})$; i.e.

$$
\vee_{k=0}^{n} s^{-k}(\widehat{\mathcal{C}})=\left\{C\left(\begin{array}{ccc}
A_{0} & \cdots & A_{n} \\
0 & \cdots & n
\end{array}\right): A_{0}, \ldots, A_{n} \in \mathcal{C}\right\}
$$

Thus, for each $n \in \mathbb{N}_{0}$, we see that

$$
\begin{aligned}
H\left(\vee_{k=0}^{n} s^{-k}(\widehat{\mathcal{C}})\right) & =\sum_{\substack{A_{k} \in \mathcal{C} \\
0 \leq k \leq n}} \eta\left(\mu^{\mathbf{X}}\left(C\left(\begin{array}{ccc}
A_{0} & \cdots & A_{n} \\
0 & \cdots & n
\end{array}\right)\right)\right) \text { by (15) } \\
& =\sum_{\substack{A_{k} \in \mathcal{C} \\
0 \leq k \leq n}} \eta\left(\mu\left(\cap_{k=0}^{n}\left(X_{k} \in A_{k}\right)\right)\right) \text { by (14) } \\
& =H\left(X_{0}^{\mathcal{C}}, \ldots, X_{n}^{\mathcal{C}}\right) \quad \text { by definition of } \mathbf{X}_{\mathcal{C}} .
\end{aligned}
$$

Hence, from Equations (111) and (3),$H\left(\mathbf{X}_{\mathcal{C}}\right)=h^{K S}(s, \widehat{\mathcal{C}})$ for each $\mathcal{C} \in \mathcal{P}_{\text {ar }}(E)$. Whenever $E$ is a discrete space and $\mathcal{A}$ is the atomic partition on $E$, since $\mathbf{X}$ can be identified with $\mathbf{X}_{\mathcal{A}}$, it follows that $H(\mathbf{X})=H\left(\mathbf{X}_{\mathcal{A}}\right)=h^{K S}(s, \widehat{\mathcal{A}})$.

An important tool for computing the KS entropy of a DS is the Kolmogorov-Sinai Theorem. First, given a $\operatorname{DS}(\Omega, \Sigma, \mu, f)$ and a partition $\mathcal{C} \in \mathcal{P}_{a r}(\Omega)$, we say that $\mathcal{C}$ is 
a generating partition for $(\Omega, \Sigma, \mu, f)$ if

$$
\sigma\left(\cup_{n=0}^{\infty} \vee_{k=0}^{n} f^{-k}(\mathcal{C})\right)=\Sigma \text {. }
$$

Notice that the definition of a generating partition does not depend on $\mu$, but for simplicity of notation we keep the full DS.

Theorem 2.15 (Kolmogorov-Sinai Theorem). Let $(\Omega, \Sigma, \mu, f)$ be a $D S$ and $\mathcal{C}, \mathcal{D} \in$ $\mathcal{P}_{a r}(\Omega)$. If $\sigma(\mathcal{D}) \subseteq \sigma\left(\cup_{n=0}^{\infty} \vee_{k=0}^{n} f^{-k}(\mathcal{C})\right)$, then

$$
h^{K S}(f, \mathcal{C}) \geq h^{K S}(f, \mathcal{D})
$$

In particular, if $\mathcal{C}$ is a generating partition and $H(\mathcal{C})<\infty$ then $h^{K S}(f)=h^{K S}(f, \mathcal{C})$.

A proof of Theorem 2.15 can be found in [9, Theorem 4.2.2].

Corollary 2.16. Let $(\Omega, \Sigma, \mu)$ be a probability space, $(E, \mathcal{E})$ a discrete measurable space, $\mathcal{A}$ the atomic partition of $E, \mathbf{X}$ an $(\Omega, E)$ stochastic process and $\left(E^{*}, \Sigma^{*}, \mu^{\mathbf{X}}, s\right)$ the symbolic dynamics of $\mathbf{X}$. Then $H(\mathbf{X})=h^{K S}(s)=h^{K S}(s, \widehat{\mathcal{A}})$ whenever $X_{0}$ has finite entropy.

Proof. From Equation (15) we see that $\widehat{\mathcal{A}}$ is a generating partition for $\left(E^{*}, \Sigma^{*}, \mu^{\mathbf{X}}, s\right)$. Then Proposition 2.14 and Theorem 2.15 give that $h^{K S}(s)=h^{K S}(s, \widehat{\mathcal{A}})=H(\mathbf{X})$, whenever $\widehat{\mathcal{A}}$ has finite entropy. Noticing that $H(\widehat{\mathcal{A}})=H\left(\mathcal{A}_{X_{0}}\right)=H\left(X_{0}\right)<\infty$, the result follows.

Remark 2.17. Notice that the results in Proposition 2.14 and Corollary 2.16 look nearly identical except that the condition $H\left(X_{0}\right)<\infty$ has been added to the latter. This assumption is necessary due to the fact that $h^{K S}(s, \widehat{\mathcal{A}})$ is defined regardless of whether $H(\widehat{\mathcal{A}})$ is finite or infinite, but is only considered in the supremum of Equation (5) when $H(\widehat{\mathcal{A}})$ is finite.

A good resource for symbolic dynamics of Markov processes is [13].

2.4. Differences between entropy rate and KS entropy. In this section we give the differences between entropy rate and KS entropy. The first thing to notice is that dynamics of a stochastic process are probabilistic in nature, whereas the dynamics of a DS are deterministic in nature. This fact will be exploited to establish the differences in the two entropies in this section and again in Section 4 to establish differences between quantum dynamical entropy and KS entropy. The following two propositions give properties of KS entropy whose analogous statements do not hold true for entropy rate. The first proposition will use the well known fact (see e.g. [9, Equation (1.3.2)] $)$ that for any probability space $(\Omega, \Sigma, \mu)$ and partition $\mathcal{C} \in \mathcal{P}_{\text {ar }}(\Omega)$, we have

$$
H(\mathcal{C}) \leq \ln |\mathcal{C}| \leq \ln |\Omega|
$$

Proposition 2.18. Let $(\Omega, \Sigma, \mu, f)$ be a DS such that $|\Omega|<\infty$. Then $h^{K S}(f)=0$.

Proof. For any partition $\mathcal{C} \in \mathcal{P}_{a r}(\Omega)$, Equation (16) gives that $H\left(\vee_{k=0}^{n-1} f^{-k}(\mathcal{C})\right) \leq$ $\ln |\Omega|$, for each $n \in \mathbb{N}$. Therefore $h^{K S}(f, \mathcal{C})=0$ for every $\mathcal{C} \in \mathcal{P}_{\text {ar }}(\Omega)$ and thus $h^{K S}(f)=0$. 
Proposition 2.19 ([9, Fact 4.1.14]). Let $(\Omega, \Sigma, \mu, f)$ be a DS. Then the KS entropy of $f$ is linear in time; i.e.

$$
h^{K S}\left(f^{n}\right)=n h^{K S}(f), \quad \text { for all } n \in \mathbb{N}_{0} .
$$

The example of a stationary Markov process governed by the unbiased random walk on a cycle (which is defined below) is enough to show that entropy rate does not have the analogous properties of KS entropy given in Propositions 2.18 and 2.19. Let $V=\{0, \ldots, N-1\}$, for some $N \in \mathbb{N}$ with $N \geq 3$, let $\mu$ be the uniform distribution on $V$ and consider the discrete probability space $(V, \mathcal{P}(V), \mu)$. The unbiased random walk on the $N$-cycle, $V$, is governed by the transition matrix $P$ with entries $p_{v+1, v}=$ $p_{v-1, v}=1 / 2$, where addition is done modulo $N$, for all $v \in V$, and $p_{u, v}=0$ if $u \neq v \pm 1$.

Proposition 2.20. Let $(V, \mathcal{P}(V), \mu)$ be the discrete probability space with $V=\{0, \ldots, N-$ $1\}$, for some $N \in \mathbb{N}$ with $N$ odd and $N \geq 3$, $\mu$ be the uniform distribution on $V$ and $P$ be the transition matrix governing the unbiased random walk on $V$. Then $H(P)=\ln 2$ and $H\left(P^{2}\right)=\frac{3}{2} \ln 2$.

Proof. Clearly $\mu$ is the unique probability measure that is $P$-invariant. Therefore Equation (13) gives that

$$
H(P)=\sum_{v \in V} \mu_{v} \sum_{u \in V} \eta\left(p_{u, v}\right)=\sum_{v \in V} \frac{1}{N} 2 \eta\left(\frac{1}{2}\right)=\ln 2 .
$$

Also notice that, for all $v \in V, P^{2}$ has entries $p_{v \pm 2, v}^{(2)}=\frac{1}{4}, p_{v, v}^{(2)}=\frac{1}{2}$ and $p_{u, v}^{(2)}=0$ in all other cases, where addition is done modulo $N$. Again $\mu$ is the unique probability measure that is $P^{2}$-invariant and Equation (13) gives that

$$
H\left(P^{2}\right)=\sum_{v \in V} \mu_{v} \sum_{u \in V} \eta\left(p_{u, v}^{(2)}\right)=\sum_{v \in V} \frac{1}{N}\left(2 \eta\left(\frac{1}{4}\right)+\eta\left(\frac{1}{2}\right)\right)=\frac{3}{2} \ln 2 .
$$

Proposition 2.20 and Corollary 2.16 establish that the KS entropy of the symbolic dynamics of a stochastic process with range in a finite measurable space need not be zero, whereas Proposition 2.18 states that the KS entropy of a finite DS must be 0. Propositions 2.20 and 2.18 do not contradict Proposition 2.14 since the cardinality of $E^{*}$, in the symbolic dynamics of a stochastic process, is not finite unless the range, $E$, of the stochastic process is a singleton. Also, Proposition 2.20 says that entropy rate is not linear in time whereas Proposition 2.19 says that KS entropy is linear in time. Again these two propositions are not contradictory. We will elaborate a bit further for clarity. In what follows, we will denote the $\mathrm{KS}$ entropy of a DS $(\Omega, \Sigma, \mu, f)$ by $h^{K S}(f, \mu)$ instead of $h^{K S}(f)$ to distinguish between different measures. We will denote the partition dependent KS entropy similarly.

Let $(V, \mathcal{P}(V), \mu)$ be the finite discrete probability space with $V=\{0, \ldots, N-1\}$, for some $N \in \mathbb{N}$ with $N$ odd and $N \geq 3, \mu$ be the uniform distribution on $V$, $P$ be the transition matrix governing the unbiased random walk on the $N$-cycle, $V, \mathbf{X}=\left(X_{n}\right)_{n=0}^{\infty}$ be any stationary Markov process governed by the transition matrix $P, \mathcal{A}_{V}$ the atomic partition of $V$ and $\left(V^{*}, \mathcal{P}(V)^{*}, \mu^{\mathbf{X}}, s_{1}\right)$ be the symbolic dynamics of $\mathbf{X}$, where $s_{1}$ denotes the shift map on $V^{*}$. Since $\widehat{\mathcal{A}_{V}}$ is a generating 
partition for $\left(V^{*}, \mathcal{P}(V)^{*}, \mu^{\mathbf{X}}, s_{1}\right)$, Corollary 2.16 shows that $H(\mathbf{X})=h^{K S}\left(s_{1}, \mu^{\mathbf{X}}\right)=$ $h^{K S}\left(s_{1}, \mu^{\mathbf{X}}, \widehat{\mathcal{A}_{V}}\right)$. Since $\widehat{\mathcal{A}_{V}} \vee s_{1}^{-1}\left(\widehat{\mathcal{A}_{V}}\right)$ is a generating partition for $\left(V^{*}, \mathcal{P}(V)^{*}, \mu^{\mathbf{X}}, s_{1}^{2}\right)$ with finite entropy, the KS Theorem gives that $h^{K S}\left(s_{1}^{2}, \mu^{\mathbf{X}}\right)=h^{K S}\left(s_{1}^{2}, \mu^{\mathbf{X}}, \widehat{\mathcal{A}_{V}} \vee\right.$ $\left.s_{1}^{-1}\left(\widehat{\mathcal{A}_{V}}\right)\right)$. Next, consider the stationary Markov process $\mathbf{Y}=\left(\left(X_{2 n}, X_{2 n+1}\right)\right)_{n=0}^{\infty}$ and let $\left((V \times V)^{*}, \mathcal{P}(V \times V)^{*}, \mu^{\mathbf{Y}}, s_{2}\right)$ be the symbolic dynamics of $\mathbf{Y}$ and $\mathcal{A}_{V \times V}$ be the atomic partition of $V \times V$, where $s_{2}$ denotes the shift map on $(V \times V)^{*}$. Since $\widehat{\mathcal{A}_{V \times V}}$ is a generating partition for $\left((V \times V)^{*}, \mathcal{P}(V \times V)^{*}, \mu^{\mathbf{Y}}, s_{2}\right)$ with finite entropy, Corollary 2.16 gives that $H(\mathbf{Y})=h^{K S}\left(s_{2}, \mu^{\mathbf{Y}}\right)=h^{K S}\left(s_{2}, \mu^{\mathbf{Y}}, \widehat{\mathcal{A}_{V \times V}}\right)$. Notice that

$$
\mu^{\mathbf{Y}}\left(C\left(\begin{array}{ccc}
\left(e_{0}, e_{1}\right) & \cdots & \left(e_{2 n}, e_{2 n+1}\right) \\
0 & \cdots & n
\end{array}\right)\right)=\mu^{\mathbf{X}}\left(C\left(\begin{array}{ccc}
e_{0} & \cdots & e_{2 n+1} \\
0 & \cdots & 2 n+1
\end{array}\right)\right),
$$

for all $e_{0}, \ldots, e_{2 n+1} \in E$. Thus

$$
\left.H_{\mu} \mathrm{Y}\left(\vee_{k=0}^{n} s_{2}^{-k}\left(\widehat{\mathcal{A}_{V \times V}}\right)\right)\right)=H_{\mu} \mathrm{x}\left(\bigvee_{k=0}^{n}\left(s_{1}^{2}\right)^{-k}\left(\widehat{\mathcal{A}_{V}} \vee s^{-1}\left(\widehat{\mathcal{A}_{V}}\right)\right) \text { for all } n \in \mathbb{N}_{0}\right.
$$

and therefore

$$
H(\mathbf{Y})=h^{K S}\left(s_{2}, \mu^{\mathbf{Y}}, \widehat{\mathcal{A}_{V \times V}}\right)=h^{K S}\left(s_{1}^{2}, \mu^{\mathbf{X}}, \widehat{\mathcal{A}_{V}} \vee s_{1}^{-1}\left(\widehat{\mathcal{A}_{V}}\right)\right)=2 H(\mathbf{X}) .
$$

In other words, the KS entropy of $\left(V^{*}, \mathcal{P}(V)^{*}, \mu^{\mathbf{X}}, s_{1}^{2}\right)$ is equal to the KS entropy of $\left((V \times V)^{*}, \mathcal{P}(V \times V)^{*}, \mu^{\mathbf{Y}}, s_{2}\right)$ and corresponds to the entropy rate of $\mathbf{Y}$.

Next consider the stochastic process $\mathbf{Z}=\left(X_{2 n}\right)_{n=0}^{\infty}$ and let $\left(V^{*}, \mathcal{P}(V)^{*}, \mu^{\mathbf{Z}}, s_{1}\right)$ be the symbolic dynamics of $\mathbf{Z}$. Then $\mathbf{Z}$ is the stationary and invariant Markov process governed by the transition matrix $P^{2}$ and, from Proposition 2.20 and Corollary 2.16, $H(\mathbf{Z})=h^{K S}\left(s_{1}, \mu^{\mathbf{Z}}\right)=h^{K S}\left(s_{1}, \mu^{\mathbf{Z}}, \widehat{\mathcal{A}_{V}}\right)=\frac{3}{2} \ln 2$. Thus Propositions 2.19 and 2.20 are not contradictory as $2 H(P)=2 H(\mathbf{X})=h^{K S}\left(s_{1}^{2}, \mu^{\mathbf{X}}\right)$ corresponds to the entropy rate of $\mathbf{Y}$, whereas $H\left(P^{2}\right)=H(\mathbf{Z})=h^{K S}\left(s_{1}, \mu^{\mathbb{Z}}\right)$ corresponds to the entropy rate of $\mathrm{Z}$.

\section{Measurements}

Here we recall the formalism of measurements, developed by Edwards [10] and Davies and Lewis [8, 7]. We follow mainly the notations of Davies and Lewis. We define phase space, state space, observables and instruments. This formalism is general enough that it holds valid for classical mechanics, Hilbert space quantum mechanics, and $C^{*}$-algebra quantum mechanics (even though we don't discuss the last one here).

A state space is defined as a pair $(X, K)$, where

(i) $X$ is a real Banach space with norm $\|\cdot\|$,

(ii) $K$ is a closed cone in $X$,

(iii) if $u, v \in X$, then $\|u\|+\|v\|=\|u+v\|$, and

(iv) if $u \in X$ and $\epsilon>0$, then there exists $u_{1}, u_{2} \in K$ such that $u=u_{1}-u_{2}$ and $\left\|u_{1}\right\|+\left\|u_{2}\right\|<\|u\|+\epsilon$.

It can be shown that, for any state space $(X, K)$, there exists a unique positive linear functional $\tau: X \rightarrow \mathbb{R}$ such that $\tau(u) \leq\|u\|$, for $u \in X$, with equality when $u \in K$. We say that $u \in K$ is a state if $\tau(u)=1$. It should be remarked that all examples of state spaces presented here will satisfy a strengthening of (iv); namely,

$\left(\right.$ iv $\left.^{\prime}\right)$ if $u \in X$, then there exists $u_{1}, u_{2} \in K$ such that $u=u_{1}-u_{2}$ and $\left\|u_{1}\right\|+\left\|u_{2}\right\|=$ $\|u\|$. 
A phase space is defined as an arbitrary measurable space $(\Omega, \Sigma)$, where $\Omega$ represents the collection of possible outcomes of a measurement and is sometimes called the value space in the literature. We say that $x: \Sigma \rightarrow X^{*}$ is an observable if, for every $E \in \Sigma, 0 \leq x(E) \leq \tau$ and $x(\Omega)=\tau$, where the partial ordering on $X^{*}$ is defined by $\phi \leq \psi$ whenever $\phi(u) \leq \psi(u)$ for all $u \in K$. Given a state $u \in K$, an observable $x$, and $E \in \Sigma$, we interpret $x(E) u$ as the probability that a system in state $u$ takes values in $E$ when observed with the observable $x$.

An operation is a positive, bounded linear operator $T: X \rightarrow X$, such that $0 \leq \tau(T u) \leq \tau(u)$ for every $u \in K$. We denote by $\mathcal{O}:=\mathcal{O}(X)$ the set of all operations on $X$. Finally, we define an instrument as a map $\mathcal{T}: \Sigma \rightarrow \mathcal{O}$ such that $\tau(\mathcal{T}(\Omega) u)=\tau(u)$, for all $u \in K$, and $\mathcal{T}\left(\cup_{n} E_{n}\right)=\sum_{n} \mathcal{T}\left(E_{n}\right)$, for any disjoint sequence of sets $\left\{E_{n}\right\} \subseteq \Sigma$, where convergence of the sum is in the strong operator topology.

Notice that for any instrument $\mathcal{T}$, one can define a unique observable $x_{\mathcal{T}}$ by setting $x_{\mathcal{T}}(E) u=\tau(\mathcal{T}(E) u)$ for $u \in X$ and $E \in \Sigma$. However, it is possible that two distinct instruments, $\mathcal{T} \neq \mathcal{S}$, give rise to the same observable, $x_{\mathcal{T}}=x_{\mathcal{S}}$. From the above correspondence, given an initial state $u \in K$ and $E \in \Sigma$, we can interpret $\mathcal{T}(E) u / x_{\mathcal{T}}(E) u \in K$ as the state of the system immediately after measuring the system in state $u$ with the instrument $\mathcal{T}$ and obtaining values in the set $E$.

The next example illustrates measurements in the standard version of classical mechanics.

Example 3.1 (Classical Mechanics). Let $\Omega$ be a locally compact Hausdorff space and $\mathcal{B}$ be the Borel $\sigma$-algebra of $\Omega$. Take $X$ to be the real Banach space of all countably additive, regular, real-valued Borel measures on $X$ and take the norm on $X$ to be the total variation norm and the closed cone, $K$, to be the set of non-negative measures in $X$. It is clear that $(X, K)$ satisfies conditions (i)-(iii) and (iv') of a state space by taking $u_{1}$ and $u_{2}$ to be the positive and negative parts, respectively, of $u \in X$ given by the Hahn decomposition. Furthermore, the linear functional $\tau$ is given by $\tau(\nu)=\int_{\Omega} d \nu=\nu(\Omega)$ for any $\nu \in X$. The phase space is given by $(\Omega, \mathcal{B})$. We define the (classical) sharp measurement instrument $\mathcal{T}$ by

$$
\mathcal{T}(E) \nu(A)=\nu(A \cap E) \quad \text { for } \nu \in X \text { and } A, E \in \mathcal{B} \text {. }
$$

The corresponding observable is given by

$$
x_{\mathcal{T}}(E) \nu=\tau(\mathcal{T}(E) \nu)=\nu(E) \quad \text { for } E \in \mathcal{B} \text { and } \nu \in X .
$$

The next example illustrates measurements in the Hilbert space formalism of quantum mechanics with discrete phase space.

Example 3.2 (Hilbert Space Quantum Mechanics). Let $H$ be a Hilbert space. Take $X=S_{1}^{s a}(H)$ to be the real Banach space of self-adjoint, trace class operators on $H$ equipped with the trace class norm and the closed cone, $K=S_{1}^{+}(H)$, to be collection of all the positive, trace class operators on $H$. It is clear that the state space $(X, K)$ satisfies conditions ( $i$ )-(iii) and (iv') of a state space by taking $u_{1}$ and $u_{2}$ to be the positive and negative parts, respectively, of $u \in X$ given by the functional calculus. Furthermore, the linear functional $\tau$ is given by the trace, $\operatorname{tr}$. Let $(\Omega, \mathcal{P}(\Omega))$ be a discrete phase space and $\left(B_{i}\right)_{i \in \Omega} \subseteq B(H)$ be a collection of bounded operators, indexed by $\Omega$, such that $\sum_{i \in \Omega} B_{i}^{*} B_{i}=\mathbb{1}$, where $\mathbb{1}$ is the identity operator on $H$. We define 
the instrument $\mathcal{T}$, given by the family $\left(B_{i}\right)_{i \in \Omega}$, by

$$
\mathcal{T}(E) \rho=\sum_{i \in E} B_{i} \rho B_{i}^{*} \quad \text { for each } \rho \in X \text { and } E \in \mathcal{P}(\Omega),
$$

where the sums are taken with respect to the strong operator topology if $\Omega$ is countably infinite. When restricted to actions on $K, \mathcal{T}$ represents a positive operator valued measure. The corresponding observable is given by

$$
x_{\mathcal{T}}(E) \rho=\sum_{i \in E} \operatorname{tr}\left(B_{i} \rho B_{i}^{*}\right) \quad \text { for each } \rho \in X \text { and } E \in \mathcal{P}(\Omega) .
$$

Whenever the family of bounded operators are pairwise orthogonal projections, we denote them by $\left(P_{i}\right)_{i \in \Omega}$ and note that $\sum_{i \in \Omega} P_{i}^{*} P_{i}=\sum_{i \in \Omega} P_{i}=\mathbb{1}$. In this case, the corresponding instrument $\mathcal{T}$, for $\left(P_{i}\right)_{i \in \Omega}$, is called a Lüders-von Neumann instrument and is given by

$$
\mathcal{T}(E) \rho=\sum_{i \in E} P_{i} \rho P_{i} \quad \text { for } \rho \in X \text { and } E \in \mathcal{P}(\Omega),
$$

where the sums are taken with respect to the strong operator topology if $\Omega$ is countably infinite. It is worth noting that $\mathcal{T}$ is defined by the "collapse of wave function formula." The corresponding observable is defined analogously.

Whenever the family $\left(P_{i}\right)_{i \in \Omega}$ consists of orthogonal, rank-1 projections, the Lüdersvon Neumann instrument $\mathcal{T}$ is called a coherent states instrument (see [21, Section IV].) In this paper whenever we refer to a coherent states instrument we will always mean a Lüders-von Neumann instrument given by a family of orthogonal, rank-1 projections as opposed to the more general definition given in [21, Example $(\mathrm{M})]$.

\section{Quantum Dynamical Entropy}

There have been many successful attempts to generalize KS entropy to a quantum dynamical entropy in [5, 2, 21, 1, 15] and more. In [21, the authors use a semiclassical approach to develop a quantum dynamical entropy using the general notions of state space, phase space, observables and instruments introduced in Section 3, Furthermore, the quantum dynamical entropy of [21] has the benefit that it is not guaranteed to be zero for finite systems, unlike the others. This is due to its symbolic dynamics approach.

Let $(X, K)$ be a state space and $u \in K$ be a state. Let $(\Omega, \Sigma)$ be a phase space, $\mathcal{T}$ an instrument and $\Theta$ a $\tau$-preserving automorphism of $X$; i.e. $\tau(\Theta v)=\tau(v)$ for all $v \in X$. Let $\left(\Omega^{*}, \Sigma^{*}\right)$ be the measurable space defined in Section 2.3 . We will define an instrument and state-dependent probability measure, $\mu^{(\Theta, \mathcal{T}, u)}$, on $\left(\Omega^{*}, \Sigma^{*}\right)$. First, we define the values of $\mu^{(\Theta, \mathcal{T}, u)}$ on the cylinder sets in $\Sigma^{*}$ with an initial interval of time sequences, $\{k\}_{k=0}^{n}$ for some $n \in \mathbb{N}_{0}$, by

$$
\mu^{(\Theta, \mathcal{T}, u)}\left(C\left(\begin{array}{ccc}
A_{0} & \cdots & A_{n} \\
0 & \cdots & n
\end{array}\right)\right)=\tau\left(\mathcal{T}\left(A_{n}\right) \circ \Theta \circ \cdots \circ \mathcal{T}\left(A_{1}\right) \circ \Theta \circ \mathcal{T}\left(A_{0}\right) u\right),
$$

for all $A_{0}, \ldots, A_{n} \in \Sigma$. Since the collection of cylinder sets with an initial interval of time sequences form a $\pi$-system which generates $\Sigma^{*}$, there is a unique extension of $\mu^{(\Theta, \mathcal{T}, u)}$ to $\left(\Omega^{*}, \Sigma^{*}\right)$ by the $\pi-\lambda$ Theorem.

Notice that, for a stochastic process $\mathbf{X}$, we defined the measure $\mu^{\mathbf{X}}$ first on cylinder sets with arbitrary time sequences (Equation (14)), whereas the measure, $\mu^{(\Theta, \mathcal{T}, u)}$ in 
Equation (18), was defined first on the cylinder sets with an initial interval of time sequences. By defining $\mu^{(\Theta, \mathcal{T}, u)}$ in this way, we have that, for $A_{0}, A_{2} \in \Sigma$,

$$
\mu^{(\Theta, \mathcal{T}, u)}\left(C\left(\begin{array}{cc}
A_{0} & A_{2} \\
0 & 2
\end{array}\right)\right)=\mu^{(\Theta, \mathcal{T}, u)}\left(C\left(\begin{array}{ccc}
A_{0} & \Omega & A_{2} \\
0 & 1 & 2
\end{array}\right)\right)=\tau\left(\mathcal{T}\left(A_{2}\right) \circ \Theta \circ \mathcal{T}(\Omega) \circ \Theta \circ \mathcal{T}\left(A_{0}\right) u\right)
$$

which is not necessarily equal to $\tau\left(\mathcal{T}\left(A_{2}\right) \circ \Theta^{2} \circ \mathcal{T}\left(A_{0}\right) u\right)$. Therefore $\mu^{(\Theta, \mathcal{T}, u)}\left(C\left(\begin{array}{cc}A_{0} & A_{2} \\ 0 & 2\end{array}\right)\right)$ is interpreted as the probability that a system in initial state $u$ will be measured at times $0,1,2$ and record the measurement sequence $\left(A_{0}, A_{2}\right)$ at times 0 and 2 . In other words, we must assume that the instrument $\mathcal{T}$ is interacting with the system at all integer times, regardless of whether or not we record a measurement.

Define the $\left(\Omega^{*}, \Omega\right)$ stochastic process $\mathbf{X}^{(\Theta, \mathcal{T}, u)}=\left(X_{n}^{(\Theta, \mathcal{T}, u)}\right)_{n=0}^{\infty}$ by $X_{n}^{(\Theta, \mathcal{T}, u)}(x)=x_{n}$ for each $x=\left(x_{m}\right)_{m \in \mathbb{N}_{0}} \in \Omega^{*}$ and, for each $\mathcal{C} \in \mathcal{P}_{a r}(\Omega)$, define the $\left(\Omega^{*}, \mathcal{C}\right)$ stochastic process $\mathbf{X}_{\mathcal{C}}^{(\Theta, \mathcal{T}, u)}=\left(X_{n}^{(\Theta, \mathcal{T}, u, \mathcal{C})}\right)_{n=0}^{\infty}$ by $X_{n}^{(\Theta, \mathcal{T}, u, \mathcal{C})}=i_{\mathcal{C}} \circ X_{n}^{(\Theta, \mathcal{T}, u)}$, where $i_{\mathcal{C}}: \Omega \rightarrow \mathcal{C}$ is the natural map that assigns to each $x \in \Omega$ the unique $A \in \mathcal{C}$ such that $x \in A$. Even though the formulas of $\mathbf{X}^{(\Theta, \mathcal{T}, u)}$ and $\mathbf{X}_{\mathcal{C}}^{(\Theta, \mathcal{T}, u)}$ do not depend on $\Theta, \mathcal{T}$ and $u$, the measure $\mu^{(\Theta, \mathcal{T}, u)}$ on their domain, $\Omega^{*}$, depends on $\Theta, \mathcal{T}$ and $u$.

We define the Słomczyński-Życzkowski (SZ) entropy of $(\Theta, \mathcal{T}, \boldsymbol{u})$ with respect to $\mathcal{C}$ to be the entropy rate of the stochastic process $\mathbf{X}_{\mathcal{C}}^{(\Theta, \mathcal{T}, u)}$. It is related to $\mu^{(\Theta, \mathcal{T}, u)}$ by the equation

$$
h^{S Z}(\Theta, \mathcal{T}, u, \mathcal{C}):=H\left(\mathbf{X}_{\mathcal{C}}^{(\Theta, \mathcal{T}, u)}\right)=\lim _{n \rightarrow \infty} \frac{1}{n} \sum_{\substack{A_{k} \in \mathcal{C} \\
0 \leq k \leq n-1}} \eta\left(\mu^{(\Theta, \mathcal{T}, u)}\left(C\left(\begin{array}{ccc}
A_{0} & \cdots & A_{n-1} \\
0 & \cdots & n-1
\end{array}\right)\right)\right),
$$

whenever the limit exists. The second equality follows from Equations (17) and (11).

Remark 4.1. Let $s$ be the shift transformation on $\left(\Omega^{*}, \Sigma^{*}, \mu^{(\Theta, \mathcal{T}, u)}\right)$ so that $\left(\Omega^{*}, \Sigma^{*}, \mu^{(\Theta, \mathcal{T}, u)}, s\right)$ is a DS. From Proposition 2.14 and the definition of $\mathbf{X}_{\mathcal{C}}^{(\Theta, \mathcal{T}, u)}$ it is clear that

$$
h^{S Z}(\Theta, \mathcal{T}, u, \mathcal{C})=H\left(\mathbf{X}_{\mathcal{C}}^{(\Theta, \mathcal{T}, u)}\right)=h^{K S}(s, \widehat{\mathcal{C}})
$$

Next we split the SZ entropy of $(\Theta, \mathcal{T}, u)$ with respect to $\mathcal{C}$ into two different causes for randomness. The first cause of randomness is that caused by the choice of instrument, is referred to as the measurement SZ entropy and is given by

$$
h_{\text {meas }}^{S Z}(\mathcal{T}, u, \mathcal{C}):=h^{S Z}(\mathbb{1}, \mathcal{T}, u, \mathcal{C}) .
$$

The second cause of randomness is given by the dynamics; i.e. the automorphism $\Theta$, is referred to as the dynamical SZ entropy, and is given by the difference

$$
h_{\text {dyn }}^{S Z}(\Theta, \mathcal{T}, u, \mathcal{C}):=h^{S Z}(\Theta, \mathcal{T}, u, \mathcal{C})-h_{\text {meas }}^{S Z}(\mathcal{T}, u, \mathcal{C}) .
$$

Finally, we define the dynamical SZ entropy of $(\Theta, \mathcal{T}, \boldsymbol{u})$ by

$$
h_{\mathrm{dyn}}^{S Z}(\Theta, \mathcal{T}, u):=\sup _{\mathcal{C} \in \mathcal{P}_{a r}(\Omega) H(\widehat{\mathcal{C}})<\infty} h_{\mathrm{dyn}}^{S Z}(\Theta, \mathcal{T}, u, \mathcal{C}) .
$$

The following lemma is claimed in [20]. For completeness we provide the proof.

Lemma 4.2. Let $(\Omega, \mathcal{P}(\Omega)),(X, K)$ and $H$ be as in Example 3.2 and let $\mathcal{T}$ be a Lüders-von Neumann instrument. Then $h_{\text {meas }}^{S Z}(\mathcal{T}, \rho, \mathcal{C})=0$ for any state $\rho \in K$ and any $\mathcal{C} \in \mathcal{P}_{\text {ar }}(\Omega)$ with finite entropy; i.e. $H(\widehat{\mathcal{C}})<\infty$. 
Proof. Let $\left(P_{i}\right)_{i \in \Omega}$ be the family of pairwise orthogonal projections that governs $\mathcal{T}$ and fix a state $\rho \in K$. Since the family, $\left(P_{i}\right)_{i \in \Omega}$, is pairwise orthogonal we have, for any $n \in \mathbb{N}_{0}$ and $A_{0}, \ldots, A_{n} \in \mathcal{P}(\Omega)$, that

$$
\mu^{(\mathbb{1}, \mathcal{T}, \rho)}\left(C\left(\begin{array}{ccc}
A_{0} & \cdots & A_{n} \\
0 & \cdots & n
\end{array}\right)\right)= \begin{cases}\sum_{a \in A_{0}} \operatorname{tr}\left(P_{a} \rho P_{a}\right)=\mu^{(\mathbb{1}, \mathcal{T}, \rho)}\left(\left(\begin{array}{c}
A_{0} \\
0
\end{array}\right)\right) & \text { if } A_{0}=\cdots=A_{n} \\
0 & \text { else }\end{cases}
$$

Therefore, for any $\mathcal{C} \in \mathcal{P}_{a r}(\Omega)$ with $H(\widehat{\mathcal{C}})<\infty$, we have

$$
\begin{aligned}
h_{\text {meas }}^{S Z}(\mathcal{T}, \rho, \mathcal{C}) & \left.=\lim _{n \rightarrow \infty} \frac{1}{n} \sum_{A \in \mathcal{C}} \eta\left(\mu^{(\mathbb{1}, \mathcal{T}, \rho)}\left(C\left(\begin{array}{l}
A \\
0
\end{array}\right)\right)\right) \quad \text { by (19) }\right) \text { and (20) } \\
& =\lim _{n \rightarrow \infty} \frac{1}{n} H(\widehat{\mathcal{C}})=0 \quad \text { by the definition of } \widehat{\mathcal{C}} .
\end{aligned}
$$

Remark 4.3. It is natural to consider only the partitions $\mathcal{C} \in \mathcal{P}_{\text {ar }}(\Omega)$ with finite entropy in Lemma 4.2. because these are the only partitions considered in Equation (21).

Fix a discrete phase space $(\Omega, \Sigma)$ with $|\Omega|=N$. Then Lemma 4.2, together with Equation (16) , implies that $h_{\mathrm{dyn}}^{S Z}(\Theta, \mathcal{T}, \rho, \mathcal{C})=h^{S Z}(\Theta, \mathcal{T}, \rho, \mathcal{C})$ for any unitary transformation $\Theta$, partition $\mathcal{C}$, state $\rho$, and coherent states instrument $\mathcal{T}$. Next we show that the measurement SZ entropy for classical sharp measurement instruments is equal to 0 as well.

Lemma 4.4. Let $(\Omega, \mathcal{B}),(X, K), \tau$ and $\mathcal{T}$ be as in Example 3.1. Then, for any state $\mu \in K$ and partition $\mathcal{C} \in \mathcal{P}_{\text {ar }}(\Omega)$ with finite entropy, we have $h_{\text {meas }}^{S Z}(\mathcal{T}, \mu, \mathcal{C})=0$.

Proof. Fix a state $\mu \in K$ and a partition $\mathcal{C} \in \mathcal{P}_{a r}(\Omega)$ with finite entropy. Then, for any $n \in \mathbb{N}_{0}$ and $A_{0}, \ldots, A_{n} \in \mathcal{C}$, we have that

$$
\mu^{(\mathbb{1}, \mathcal{T}, \mu)}\left(C\left(\begin{array}{ccc}
A_{0} & \cdots & A_{n} \\
0 & \cdots & n
\end{array}\right)\right)= \begin{cases}\mu\left(A_{0}\right) & \text { if } A_{0}=\cdots=A_{n} \\
0 & \text { else }\end{cases}
$$

Therefore

$$
\begin{aligned}
h_{\text {meas }}^{S Z}(\mathcal{T}, \mu, \mathcal{C}) & =\lim _{n \rightarrow \infty} \frac{1}{n} \sum_{A \in \mathcal{C}} \eta(\mu(A)) \\
& =\lim _{n \rightarrow \infty} \frac{1}{n} H(\mathcal{C})=0 .
\end{aligned}
$$

The following result is claimed without proof in [21, Proposition 4(A)] and states that the KS and SZ entropies agree for classical dynamical systems with sharp instruments.

Proposition 4.5. Let $(\Omega, \mathcal{B}),(X, K), \tau$ and $\mathcal{T}$ be as in Example 3.1. Let $\mu \in K$ be a state; i.e. a probability measure on $(\Omega, \mathcal{B})$, and $f: \Omega \rightarrow \Omega$ a measurable map so that $(\Omega, \mathcal{B}, \mu, f)$ is a $D S$. Let $T_{f}: X \rightarrow X$ be the automorphism known as the Koopman operator defined by

$$
T_{f}(\nu)(A):=\nu\left(f^{-1}(A)\right) \text { for all } \nu \in X \text { and } A \in \mathcal{B} \text {. }
$$

Then for each $\mathcal{C} \in \mathcal{P}_{a r}(\Omega), h^{K S}(f, \mathcal{C})=h_{d y n}^{S Z}\left(T_{f}, \mathcal{T}, \mu, \mathcal{C}\right)$. 
Proof. Fix a partition $\mathcal{C} \in \mathcal{P}_{a r}(\Omega)$. For all $n \in \mathbb{N}_{0}$ and $A_{0}, \ldots, A_{n} \in \mathcal{C}$ we see that

$$
\begin{aligned}
\mu^{\left(T_{f}, \mathcal{T}, \mu\right)}\left(C\left(\begin{array}{ccc}
A_{0} & \cdots & A_{n} \\
0 & \cdots & n
\end{array}\right)\right) & =\tau\left(\mathcal{T}\left(A_{n}\right) \circ T_{f} \circ \cdots \circ T_{f} \circ \mathcal{T}\left(A_{0}\right) \mu\right) \quad \text { by (18) } \\
& =\left(\mathcal{T}\left(A_{n}\right) \circ T_{f} \circ \cdots \circ T_{f} \circ \mathcal{T}\left(A_{0}\right)\right) \mu(X) \quad \text { by definition of } \tau \\
& =\left(\mathcal{T}\left(A_{n}\right) \circ T_{f} \circ \cdots \circ \mathcal{T}\left(A_{1}\right) \circ T_{f}\right) \mu\left(A_{0}\right) \quad \text { by (17) } \\
& =\left(\mathcal{T}\left(A_{n}\right) \circ T_{f} \circ \cdots \circ T_{f} \circ \mathcal{T}\left(A_{1}\right)\right) \mu\left(f^{-1}\left(A_{0}\right)\right) \quad \text { by (??) } \\
& =\cdots \\
& =\mu\left(A_{i_{n}} \cap f^{-1}\left(A_{i_{1}}\right) \cap \cdots \cap f^{-n}\left(A_{i_{0}}\right)\right) .
\end{aligned}
$$

Using Remark 2.4 and Lemma 4.4, we get

$$
h^{K S}(f, \mathcal{C})=h^{S Z}\left(T_{f}, \mathcal{T}, \mu, \mathcal{C}\right)=h_{\mathrm{dyn}}^{S Z}\left(T_{f}, \mathcal{T}, \mu, \mathcal{C}\right)
$$

Next we examine the properties of SZ entropy for coherent states instruments. Let $H$ be a Hilbert space and $(X, K)$ the state space defined in Example 3.2, Given a unitary operator, $U$, on $H$, the unitary transformation, $\Theta: \boldsymbol{X} \rightarrow \boldsymbol{X}$, of $\boldsymbol{U}$ is given by

$$
\Theta(\cdot)=U \cdot U^{*}
$$

The following lemma gives a simplification of Equation (18) for coherent states instruments. The moreover statement of the following lemma is mentioned in [20, page 3] without proof.

Lemma 4.6. Let $H,(X, K), \tau$ and $(\Omega, \mathcal{P}(\Omega))$ be as in Example 3.2. Let $\left(P_{i}\right)_{i \in \Omega}$ be a family of orthogonal, rank-1 projections on $H$ such that $\sum_{i \in \Omega} P_{i}=\mathbb{1}$, and let $a_{i} \in H$ such that $P_{i}=\left|a_{i}\right\rangle\left\langle a_{i}\right|$, for each $i \in \Omega$. Let $\mathcal{T}$ be the coherent states instrument for $\left(P_{i}\right)_{i \in \Omega}, U$ a unitary operator on $H, \Theta$ the unitary transformation of $U$ and $\rho \in K a$ state. Then, for all $n \in \mathbb{N}_{0}$ and $A_{0}, \ldots, A_{n} \in \mathcal{P}(\Omega)$,

$$
\mu^{(\Theta, \mathcal{T}, \rho)}\left(C\left(\begin{array}{ccc}
A_{0} & \cdots & A_{n} \\
0 & \cdots & n
\end{array}\right)\right)=\sum_{\substack{a_{k} \in A_{k} \\
0 \leq k \leq n}}\left\langle a_{0}|\rho| a_{0}\right\rangle \prod_{k=1}^{n}\left|\left\langle a_{k}|U| a_{k-1}\right\rangle\right|^{2} .
$$

Moreover, $\mathbf{X}^{(\Theta, \mathcal{T}, \rho)}$ is a Markov process governed by the transition matrix $P$ on $\Omega$ with $(i, j)$-entry given by $\left|\left\langle a_{i}|U| a_{j}\right\rangle\right|^{2}$, for all $i, j \in \Omega$.

Proof. By direct calculation, Equation (18) simplifies to

$$
\begin{aligned}
\mu^{(\Theta, \mathcal{T}, \rho)}\left(C\left(\begin{array}{ccc}
A_{0} & \cdots & A_{n} \\
0 & \cdots & n
\end{array}\right)\right) & =\tau\left(\mathcal{T}\left(A_{n}\right) \circ \Theta \circ \cdots \circ \Theta \circ \mathcal{T}\left(A_{0}\right) \rho\right) \\
& =\sum_{\substack{a_{k} \in A_{k} \\
0 \leq k \leq n}} \operatorname{tr}\left(\mathcal{T}\left(\left\{a_{n}\right\}\right) \circ \Theta \circ \cdots \circ \Theta \circ \mathcal{T}\left(\left\{a_{0}\right\}\right) \rho\right) \\
& =\sum_{\substack{a_{k} \in A_{k} \\
0 \leq k \leq n}} \operatorname{tr}\left(P_{a_{n}} U \cdots U P_{a_{0}} \rho P_{a_{0}} U^{*} \cdots U^{*} P_{a_{n}}\right) \\
& =\sum_{\substack{a_{k} \in A_{k} \\
0 \leq k \leq n}} \operatorname{tr}\left(\left|a_{n}\right\rangle\left\langle a_{n}|U \cdots U| a_{0}\right\rangle\left\langle a_{0}|\rho| a_{0}\right\rangle\left\langle a_{0}\left|U^{*} \cdots U^{*}\right| a_{n}\right\rangle\left\langle a_{n}\right|\right)
\end{aligned}
$$




$$
=\sum_{\substack{a_{k} \in A_{k} \\ 0 \leq k \leq n}}\left\langle a_{0}|\rho| a_{0}\right\rangle \prod_{k=1}^{n}\left|\left\langle a_{k}|U| a_{k-1}\right\rangle\right|^{2},
$$

where the second to last equality follows from writing $P_{a_{k}}=\left|a_{k}\right\rangle\left\langle a_{k}\right|$, for all $0 \leq k \leq n$ and the last equality follows since $\overline{\left\langle a_{k-1}\left|U^{*}\right| a_{k}\right\rangle}=\left\langle a_{k}|U| a_{k-1}\right\rangle$, for all $1 \leq k \leq n$. It is immediately clear that $\mathbf{X}^{(\Theta, \mathcal{T}, \rho)}$ is a stationary Markov process governed by the transition matrix $P$.

It is worth noting that Equation (22) is a simplification of the probabilities in [21, Equations (27)-(29)] for Lüders-von Neumann coherent states instruments.

Corollary 4.7. Let $H,(X, K), \tau$ and $(\Omega, \mathcal{P}(\Omega))$ be as in Example 3.Q. Let $\left(P_{i}\right)_{i \in \Omega}$ be a family of orthogonal, rank-1 projections on $H$ such that $\sum_{i \in \Omega} P_{i}=\mathbb{1}$ and $P_{i}=$ $\left|a_{i}\right\rangle\left\langle a_{i}\right|$ for some $a_{i} \in H$ and each $i \in \Omega, \mathcal{T}$ the coherent states instrument for $\left(P_{i}\right)_{i \in \Omega}$, $U$ a unitary operator on $H, \Theta$ the unitary transformation of $U, \rho \in K$ a state and $P$ the transition matrix defined in Lemma 4.6. Then

$$
h^{S Z}(\Theta, \mathcal{T}, \rho, \mathcal{A})=\lim _{n \rightarrow \infty} \sum_{y \in \Omega}\left(P^{n} \mu\right)_{y} \sum_{x \in \Omega} \eta\left(\left|\left\langle a_{x}|U| a_{y}\right\rangle\right|^{2}\right),
$$

where $\mu=p_{X_{0}^{(\Theta, \mathcal{T}, \rho)}}$ and $\mathcal{A}$ is the atomic partition of $\Omega$. Moreover, whenever $\mu=$ $\left(\mu_{y}\right)_{y \in \Omega}$ is $P$-invariant, we have $h^{S Z}(\Theta, \mathcal{T}, \rho, \mathcal{A})=\sum_{y \in \Omega} \mu_{y} \sum_{x \in \Omega} \eta\left(\left|\left\langle a_{x}|U| a_{y}\right\rangle\right|^{2}\right)$.

Proof. This follows immediately from Lemma 4.6 and Theorem 2.11 .

In [21, Section IV] the authors require that the state $\rho$ is invariant in the sense that

$$
\Theta(\mathcal{T}(\Omega) \rho)=\rho
$$

when defining SZ entropy for coherent states instruments. This seems to be due to the fact that, in [21, Proposition 2(B)], the authors show that, under Assumption (23), for a general coherent states instrument, the stochastic process $X^{(\Theta, \mathcal{T}, \rho)}$ is stationary and hence, by the "moreover" part of Corollary 4.7, $h^{S Z}(\Theta, \mathcal{T}, \rho, \mathcal{A})=$ $\sum_{y \in \Omega} \mu_{y} \sum_{x \in \Omega} \eta\left(\left|\left\langle a_{x}|U| a_{y}\right\rangle\right|^{2}\right)$. We find Assumption (23) restrictive and do not adopt it here. It is also worth mentioning that another invariance condition often imposed on quantum dynamical systems is that $\Theta(\rho)=\rho$. For instance, for AFL entropy in [2, Page 76] which is formulated for general $C^{*}$-algebras, the authors require that a state $\omega$ satisfies $\omega \circ \Theta=\omega$ which is equivalent to $\Theta(\rho)=\rho$ in the Hilbert space quantum mechanics picture whenever $\omega$ is defined by $\omega(\cdot)=\operatorname{tr}(\rho \cdot)$. Also, given a Hilbert space $H$, a unitary operator $U$ on $H$ and a norm-1 eigenvector, $x \in H$, of $U$, the pure (or vector) state $\rho=|x\rangle\langle x|$ satisfies $\Theta(\rho)=\rho$, where $\Theta$ is the unitary transformation of $U$. There has been a lot of interest in finding these pure, invariant states in the literature for unitary quantum random walks (see e.g. [14, 11]). Therefore $\Theta(\rho)=\rho$ seems another natural definition of invariance. However, we will show in Proposition 6.1 that $\Theta(\rho)=\rho$ does not imply that $X^{(\Theta, \mathcal{T}, \rho)}$ is an invariant stochastic process.

The following result states that SZ entropy is not linear in the time interval between successive measurements which answers an open problem posed in [21, page 5692 Question (2)]. This result is in contrast to KS entropy which is linear in time 
(see Proposition 2.19). Moreover, since entropy rate is nonlinear in time (see Proposition 2.20), the result gives further evidence that measurements of a deterministic quantum system produce properties that are probabilistic in nature.

Theorem 4.8. Let $(X, K)$ be as in Example 3.2. Let $(\Omega, \mathcal{P}(\Omega))$ be a discrete phase space with $|\Omega|=N$ for some $N \in \mathbb{N}, \mathcal{T}$ a Lüders-von Neumann instrument, $\Theta$ a unitary transformation and $\rho \in K$ a state. Then $h_{d y n}^{S Z}\left(\Theta^{n}, \mathcal{T}, \rho\right) \leq N$ for all $n \in \mathbb{N}$. Therefore, if $h_{d y n}^{S Z}(\Theta, \mathcal{T}, \rho) \neq 0$, then $h_{d y n}^{S Z}\left(\Theta^{n}, \mathcal{T}, \rho\right) \neq n h_{d y n}^{S Z}(\Theta, \mathcal{T}, \rho)$ for all sufficiently large $n \in \mathbb{N}$.

Proof. Let $\left(\Omega^{*}, \mathcal{P}(\Omega)^{*}, \mu^{\left(\Theta^{n}, \mathcal{T}, \rho\right)}, s\right)$ be the symbolic dynamics of $\left(\Theta^{n}, \mathcal{T}, \rho\right)$ for each $n \in \mathbb{N}$ and let $\mathcal{A}$ be the atomic partition of $\Omega$. Using Equation (16), we have that $H_{\mu\left(\Theta^{n}, \mathcal{T}, \rho\right)}\left(\vee_{k=0}^{m-1} s^{-k}(\widehat{\mathcal{A}})\right) \leq \ln \left(\left|\vee_{k=0}^{m-1} s^{-k}(\widehat{\mathcal{A}})\right|\right) \leq m \ln N$, for all $n, m \in \mathbb{N}$. Therefore $h_{\mathrm{dyn}}^{S Z}\left(\Theta^{n}, \mathcal{T}, \rho, \mathcal{A}\right) \leq \ln N$, for each $n \in \mathbb{N}$. If $h_{\mathrm{dyn}}^{S Z}(\Theta, \mathcal{T}, \rho, \mathcal{A})=k \neq 0$, then, since $\widehat{\mathcal{A}}$ is a generating partition for $\left(\Omega^{*}, \mathcal{P}(\Omega)^{*}, \mu^{\left(\Theta^{n}, \mathcal{T}, \rho\right)}, s\right)$, we have, for all $n>\frac{\ln N}{k}$, that

$$
h_{\text {dyn }}^{S Z}\left(\Theta^{n}, \mathcal{T}, \rho\right)=h_{\text {dyn }}^{S Z}\left(\Theta^{n}, \mathcal{T}, \rho, \mathcal{A}\right) \leq \ln N<n h_{\text {dyn }}^{S Z}(\Theta, \mathcal{T}, \rho, \mathcal{A})=n h_{\text {dyn }}^{S Z}(\Theta, \mathcal{T}, \rho) .
$$

In [20] the authors establish a class of instruments which have positive dynamical SZ entropy and we give further such examples in Section 6. Therefore Proposition 4.8 does establish the nonlinearity of dynamical SZ entropy in time. We illustrate this fact in Section 6 by calculating the SZ entropy of the Hadamard walk with a Lüdersvon Neumann instrument given by a family of rank-2 projections (Theorem 6.3). The following section is dedicated to defining unitary quantum random walks and, in particular, the Hadamard walk.

\section{Unitary Quantum Random Walks}

The unitary quantum random walk (UQRW) is one of the many adaptations of the classical random walk to the quantum domain and, in particular, is the adaptation of classical random walks for closed quantum systems. We will define the UQRW on a finite or countably infinite vertex set $V$. To consider a collection of vertices in the quantum domain, Hilbert space quantum mechanics is used (see Example 3.2) and we consider the position space, $H_{P}:=\ell_{2}(V)$, with an orthonormal basis, $\{|v\rangle\}_{v \in V}$, indexed by $V$. To add internal degrees of freedom to the vertices, the coin space $H_{C}$ is used, which is an at most countably dimensional Hilbert space. In general, a UQRW is given by the unitary transformation over the tensored Hilbert space $H=H_{C} \otimes H_{P}$. The most common UQRWs are the so-call coined UQRWs. To define these we must first fix an orthonormal basis, $\{|c, v\rangle\}_{(c, v) \in C \times V}$ on $H$, sometimes referred to as the computational basis, for some index set $C$, where $|c, v\rangle=|c\rangle \otimes|v\rangle$. We say that a UQRW is coined if it is the unitary transformation of an operator $U$ of the form

$$
U=S\left(\sum_{v \in V} U_{v} \otimes|v\rangle\langle v|\right)
$$

where $U_{v}$ is a unitary operator on $H_{C}$ for each $v \in V$, and $S$ is a permutation operator which is referred to as the shift operator. By a "permutation operator", we mean 
that $S$ has the form

$$
S=\sum_{(c, v) \in C \times V}|\sigma(c, v)\rangle\langle c, v|,
$$

for some permutation $\sigma$ of $C \times V$. For each $v \in V$, the unitary operator $U_{v}$, referred to as the coin operator at $v$, changes the coin state at the vertex $v$ in a deterministic way while the shift operator $S$ moves the random walker from one site to another. In the sequel we will only consider coined UQRWs and we will drop the adjective. We say that a UQRW is space homogeneous if there exists a unitary operator $W$ on $H_{C}$ such that $W=U_{v}$ for every $v \in V$. For a space homogeneous UQRW the form of $U$ in Equation (24) simplifies to

$$
U=S\left(W \otimes \mathbb{1}_{H_{P}}\right) .
$$

We say that a shift operator $S$ is coin preserving if, for each $c \in C$, there exists a permutation $\sigma_{c}$ of $V$ such that

$$
S=\sum_{(c, v) \in C \times V}\left|c, \sigma_{c}(v)\right\rangle\langle c, v| .
$$

Furthermore, we say that a UQRW is coin preserving if its shift operator is coin preserving. Notice that a coin preserving shift operator moves the random walker from site to site without affecting the internal state of the walker.

Let $U$ be a unitary operator of the form Equation (24) with shift operator, $S$, given by Equation (25). To draw a connection to classical random walks it is helpful to visualize a random walker on the directed graph $G=(V, E)$ where $E$ is the edge set determined by the shift operator $S$. That is to say, for all $u, v \in V,(u, v) \in E$ if and only if there exists $c_{1}, c_{2} \in C$ such that $\sigma\left(c_{1}, u\right)=\left(c_{2}, v\right)$, where $\sigma$ is the map appearing in Equation (25), or, equivalently, $P_{v} S P_{u} \neq 0$, where $P_{v}=\mathbb{1}_{H_{C}} \otimes|v\rangle\langle v|$; i.e. $P_{v}$ is the projection from $H$ to $H_{C} \otimes \operatorname{span}(v)$.

We are specifically interested in the Hadamard walk, which has been studied extensively in the literature (e.g. [3, 12, 17]) and is defined below. Consider the vertex set $V=\{0, \ldots, N-1\}$, for some $N \in \mathbb{N}$ with $N \geq 2$, and set $H_{P}=\mathbb{C}^{N}$. Let $H_{C}=\mathbb{C}^{2}$, with orthonormal basis $\{|R\rangle,|L\rangle\}$. Define the (coin preserving) integer shift operator by

$$
S=\sum_{n=0}^{N-1}|R, n+1\rangle\langle R, n|+| L, n-1\rangle\langle L, n|,
$$

where addition on the integers is done modulo $N$. Throughout the rest of the paper addition (on $V$ ) will be done modulo $N$. Notice that $|R\rangle$ now corresponds to a shift right on the integers and $|L\rangle$ corresponds to a shift left on the integers. In this case the directed graph $G=(V, E)$ has edge set which is given by $E=\{(n, n+1),(n, n-$ 1) $\}_{n=0}^{N-1}$. The unitary operator

$$
h:=\frac{1}{\sqrt{2}}\left[\begin{array}{cc}
1 & 1 \\
1 & -1
\end{array}\right]
$$

on $H_{C}$ is referred to as the Hadamard matrix (or Hadamard coin/gate). The Hadamard walk on $\boldsymbol{V}$ is the map $\Theta: X \rightarrow X$, where $(X, K)$ is the state space defined in Example 3.2, given by

$$
\Theta(\rho)=U \rho U^{*}, \text { for each } \rho \in X, \text { where } U=S\left(h \otimes \mathbb{1}_{H_{P}}\right) .
$$


It is clear that the Hadamard walk is coin preserving and space homogeneous.

Remark 5.1. The Hadamard walk can easily be extended to $V=\mathbb{Z}$ as opposed to the finite cycle $V=\{0, \ldots, N-1\}$ and it is often viewed in this manner. (e.g. [17, Section 5.1])

\section{SZ ENTROpy of THE HADAMARD WALK}

Let $H=H_{C} \otimes H_{P}, C=\{R, L\}$ and take the phase space to be $(C \times V, \mathcal{P}(C \times V))$. Take $(X, K)$ be the state space $\left(S_{1}^{s a}(H), S_{1}^{+}(H)\right)$ as in Example 3.2. Define the family of orthogonal, rank-1 projections $\left(P_{e}\right)_{e \in C \times V}$ by

$$
P_{e}=|c, v\rangle\langle c, v|, \text { whenever } e=(c, v) \in C \times V .
$$

Let $\mathcal{T}$ be the coherent states instrument governed by the family $\left(P_{e}\right)_{e \in C \times V}$. The next proposition states that, for a unitary transformation $\Theta$ and a state $\rho \in K, \Theta(\rho)=\rho$ does not imply that the associated Markov chain is stationary. The result shows that this natural definition of invariance for $\rho$ is not sufficient for stationarity, whereas Assumption (23), imposed by the authors of [21], does guarantee stationarity.

Proposition 6.1. Let $\Theta$ be the Hadamard walk on $V$ with $|V|=N \geq 2$. Let $\mathcal{T}$ be the coherent states instrument given by the family of orthogonal projections $\left(P_{e}\right)_{e \in C \times V}$ and $\mathcal{A}$ be the atomic partition on $C \times V$. Let $|x\rangle=\frac{1}{\sqrt{N(4+2 \sqrt{2})}}((1+\sqrt{2})|R\rangle+|L\rangle) \otimes \sum_{v \in V}|v\rangle$ (which is a unit norm eigenvector for the unitary matrix, $U$, of the Hadamard walk) and $\rho=|x\rangle\langle x|$. Then the pmf, $p_{X_{0}^{(\Theta, \mathcal{T}, \rho)}}$, of $X_{0}^{(\Theta, \mathcal{T}, \rho)}$ is not $P$-invariant, where $P$ is the transition matrix defined in Lemma 4.6. Furthermore, the dynamical SZ entropy is equal to $h_{d y n}^{S Z}(\Theta, \mathcal{T}, \rho)=\ln 2$.

Proof. For each $(c, v) \in C \times V$,

$$
p_{X_{0}^{(\Theta, \mathcal{T}, \rho)}}(c, v)=\langle c, v|\rho| c, v\rangle=\frac{1}{N(4+2 \sqrt{2})}\left((3+2 \sqrt{2}) \delta_{c, R}+\delta_{c, L}\right) .
$$

Also, for each $e=(c, v), f=(d, u) \in C \times V$, a straightforward calculation yields

$$
\begin{aligned}
|\langle e|U| f\rangle|^{2} & = \begin{cases}\frac{1}{2} & c=R \text { and } u=v-1 \\
\frac{1}{2} & c=L \text { and } u=v+1 \\
0 & \text { else }\end{cases} \\
& =\frac{1}{2} \delta_{u, v-(-1)^{\delta_{c, L}}} .
\end{aligned}
$$

Recall that $|\langle e|U| f\rangle|^{2}$ is the $(e, f)$-entry of $P$, for each $e, f \in C \times V$. Thus, for each $e=(c, v) \in C \times V$,

$$
\begin{aligned}
\left(P p_{\left.X_{0}^{(\Theta, \mathcal{T}, \rho)}\right)_{e}}\right. & =\sum_{f \in C \times V} p_{X_{0}^{(\Theta, \mathcal{T}, \rho)}}(f)|\langle e|U| f\rangle|^{2} \\
& =\frac{1}{2}\left(p_{X_{0}^{(\Theta, \mathcal{T}, \rho)}}\left(R, v-(-1)^{\delta_{c, L}}\right)+p_{X_{0}^{(\Theta, \mathcal{T}, \rho)}}\left(L, v-(-1)^{\delta_{c, L}}\right)\right) \quad \text { by (29) } \\
& =\frac{1}{2}\left(\frac{3+2 \sqrt{2}}{N(4+2 \sqrt{2})}+\frac{1}{N(4+2 \sqrt{2})}\right)=\frac{1}{2 N} . \quad \text { by (28) }
\end{aligned}
$$


Therefore $P p_{X_{0}^{(\Theta, \mathcal{T}, \rho)}} \neq p_{X_{0}^{(\Theta, \mathcal{T}, \rho)}}$ and thus $\mathbf{X}^{(\Theta, \mathcal{T}, \rho)}$ is not stationary. Continuing to find the dynamical SZ entropy, we see that $P p_{X_{0}^{(\Theta, \tau, \rho)}}$ is the uniform distribution, $\mu$, on $C \times V$, which is invariant with respect to $P$. Thus Corollary 4.7 and Lemma 4.2 imply that

$$
h_{\mathrm{dyn}}^{S Z}(\Theta, \mathcal{T}, \rho, \mathcal{A})=\sum_{f \in C \times V} \mu_{f} \sum_{e \in C \times V} \eta\left(|\langle e|U| f\rangle|^{2}\right)=\sum_{f \in C \times V} \frac{1}{2 N} 2 \eta\left(\frac{1}{2}\right)=\ln 2,
$$

which is equal to $h_{\mathrm{dyn}}^{S Z}(\Theta, \mathcal{T}, \rho)$ because $\widehat{\mathcal{A}}$ is a generating partition for $\left(\Omega^{*}, \mathcal{P}(\Omega)^{*}, \mu^{(\Theta, \mathcal{T}, \rho)}, s\right)$ by Corollary 2.16.

As the UQRW is a quantum analogue of the classical random walk, it is natural to consider measurements of the position space only. There are two options for how to go about this. One option is to take the phase space to be $(C \times V, \mathcal{P}(C \times V))$, the coherent states instrument $\mathcal{T}$ to be given by the family $\left(P_{e}\right)_{e \in C \times V}$, defined in Equation (27), and calculate the dynamical SZ entropy with respect to the partition

$$
\mathcal{C}_{V}=\left\{C_{v}\right\}_{v \in V}, \text { where } C_{v}:=\{|R, v\rangle,|L, v\rangle\} \text {, for each } v \in V .
$$

On the other hand we could take the phase space to be $(V, \mathcal{P}(V))$, define the projections

$$
P_{v}=\mathbb{1}_{H_{C}} \otimes|v\rangle\langle v|, \text { for each } v \in V,
$$

and calculate the dynamical SZ entropy of the Lüders-von Neumann instrument $\mathcal{V}$, governed by the family $\left(P_{v}\right)_{v \in V}$, with respect to the atomic partition of $V$. We will calculate the entropies for both these scenarios (with the same initial state) on the Hadamard walk, $\Theta$, and its square, $\Theta^{2}$. We will see that the two interpretations do not yield the same entropy. This is further evidence to the sensitivity of a closed quantum system to measurement. Furthermore, Theorem 6.3 provides a concrete example illustrating the fact that dynamical SZ entropy is not linear in time.

Proposition 6.2. Let $\Theta$ be the the Hadamard walk on $V$ with $|V|=N \geq 3$. Let $\mathcal{T}$ be the coherent states instrument given by the family of orthogonal projections $\left(P_{e}\right)_{e \in C \times V}$ given in Equation (27), $\rho=\frac{\mathbb{1}_{H}}{2 N}$ and $\mathcal{C}_{V}$ the partition given in Equation (30). Then $h_{d y n}^{S Z}\left(\Theta, \mathcal{T}, \rho, \mathcal{C}_{V}\right)=\ln 2$ and $h_{d y n}^{S Z}\left(\Theta^{2}, \mathcal{T}, \rho, \mathcal{C}_{V}\right)=\frac{3}{2} \ln 2$.

Proof. Notice that $p_{X_{0}^{(\Theta, \tau, \rho)}}(c, v)=\langle c, v|\rho| c, v\rangle=\frac{1}{2 N}$ for all $(c, v) \in C \times V$ and recall that the transition matrix $P$, which governs $\Theta$ with respect to the coherent states instrument $\mathcal{T}$, has $(e, f)$-entry given by Equation (29), for every $e, f \in C \times V$. In the following, it will be more convenient to rewrite Equation (29) viewing $f$ as the fixed index. In this manner, for each $f=(c, v) \in C \times V$, we have

$$
U|f\rangle=\frac{1}{\sqrt{2}}\left(|R, v+1\rangle+(-1)^{\delta_{c, L}}|L, v-1\rangle\right),
$$

and hence

$$
|\langle e|U| f\rangle|^{2}=\frac{1}{2}\left(\delta_{e,(R, v+1)}+\delta_{e,(L, v-1)}\right) .
$$


Also, we have

$$
\begin{aligned}
\mu^{(\Theta, \mathcal{T}, \rho)}\left(C\left(\begin{array}{ccc}
C_{v_{0}} \cdots & C_{v_{n}} \\
0 & \cdots & n
\end{array}\right)\right) & =\sum_{\substack{c_{k} \in\{R, L\} \\
0 \leq k \leq n}}\left\langle c_{0}, v_{0}|\rho| c_{0}, v_{0}\right\rangle \prod_{k=1}^{n}\left|\left\langle c_{k}, v_{k}|U| c_{k-1}, v_{k-1}\right\rangle\right|^{2} \quad \text { by (22) } \\
& =\sum_{\substack{c_{k} \in\{R, L\} \\
0 \leq k \leq n}} \frac{1}{2 N} \prod_{k=1}^{n}\left(\frac{\delta_{v_{k}, v_{k-1}+1} \delta_{c_{k}, R}+\delta_{v_{k}, v_{k-1}-1} \delta_{c_{k}, L}}{2}\right) \text { by (33) } \\
& =\frac{1}{N} \prod_{k=1}^{n}\left(\frac{\delta_{v_{k}, v_{k-1}+1}+\delta_{v_{k}, v_{k-1}-1}}{2}\right)
\end{aligned}
$$

for all $v_{0}, \ldots, v_{n} \in V$, which are exactly the probabilities $p_{\mathbf{X}}\left(v_{0}, \ldots, v_{n}\right)$ of a stationary Markov chain $\mathbf{X}$ which is governed by the transition matrix, $Q$, for the unbiased random walk on the $N$-cycle $V$. Therefore $h_{\text {dyn }}^{S Z}\left(\Theta, \mathcal{T}, \rho, \mathcal{C}_{V}\right)=H(Q)=\ln 2$, where the second equality follows from Proposition 2.20 .

Next we show that $h_{\text {dyn }}^{S Z}\left(\Theta^{2}, \mathcal{T}, \rho, \mathcal{C}_{V}\right)=\frac{3}{2} \ln 2$. For all $f=(c, v) \in C \times V$, we have

$$
\begin{aligned}
U^{2}|f\rangle & =\frac{1}{\sqrt{2}} U\left(|R, v+1\rangle+(-1)^{\delta_{c, L}}|L, v-1\rangle\right) \quad \text { by (32) } \\
& =\frac{1}{2}\left((-1)^{\delta_{R, c}}|L, v-2\rangle+(-1)^{\delta_{L, c}}|R, v\rangle+|L, v\rangle+|R, v+2\rangle\right)
\end{aligned}
$$

and hence

$$
\left|\left\langle e\left|U^{2}\right| f\right\rangle\right|^{2}=\left\{\begin{array}{ll}
\frac{1}{4} & e=(R, v) \text { or }(L, v) \\
\frac{1}{4} & e=(R, v+2) \\
\frac{1}{4} & e=(L, v-2) \\
0 & \text { else }
\end{array} .\right.
$$

Notice that $\left|\left\langle e\left|U^{2}\right| f\right\rangle\right|^{2}$ in Equation (35) does not depend on the coin space component of $f$. Therefore

$$
\begin{aligned}
& \mu^{\left(\Theta^{2}, \mathcal{T}, \rho\right)}\left(C\left(\begin{array}{ccc}
C_{v_{0}} \cdots & C_{v_{n}} \\
0 & \cdots & n
\end{array}\right)\right) \\
&=\sum_{\substack{c_{k} \in\{R, L\} \\
0 \leq k \leq n}}\left\langle c_{0}, v_{0}|\rho| c_{0}, v_{0}\right\rangle \prod_{k=1}^{n}\left|\left\langle c_{k}, v_{k}\left|U^{2}\right| c_{k-1}, v_{k-1}\right\rangle\right|^{2} \quad \text { by (22) } \\
&=\sum_{\substack{c_{k} \in\{R, L\} \\
0 \leq k \leq n}} \frac{1}{2 N} \prod_{k=1}^{n}\left(\frac{\delta_{c_{k}, L} \delta_{v_{k}, v_{k-1}-2}+\delta_{c_{k}, L} \delta_{v_{k}, v_{k-1}}+\delta_{c_{k}, R} \delta_{v_{k}, v_{k-1}}+\delta_{c_{k}, R} \delta_{v_{k}, v_{k-1}+2}}{4}\right) \quad \text { by (邽5) } \\
&= \frac{1}{N} \prod_{k=1}^{n}\left(\frac{1}{4} \delta_{v_{k}, v_{k-1}-2}+\frac{1}{2} \delta_{v_{k}, v_{k-1}}+\frac{1}{4} \delta_{v_{k}, v_{k-1}+2}\right)
\end{aligned}
$$

for all $v_{0}, \ldots, v_{n} \in V$, which are exactly the probabilities $p_{\mathbf{Y}}\left(v_{0}, \ldots, v_{n}\right)$ of a stationary Markov chain $\mathbf{Y}$ which is governed by the transition matrix $Q^{2}$. Therefore $h_{\text {dyn }}^{S Z}\left(\Theta^{2}, \mathcal{T}, \rho, \mathcal{C}_{V}\right)=H\left(Q^{2}\right)=\frac{3}{2} \ln 2$, where the second equality follows from Proposition 2.20 . 
Theorem 6.3. Let $\Theta$ be the Hadamard walk on $V$ with $|V|=N \geq 3$. Let $\mathcal{V}$ be the Lüders-von Neumann instrument given by the family of orthogonal rank-2 projections $\left(P_{v}\right)_{v \in V}$ defined in Equation (31) and $\rho=\frac{\mathbb{1}_{H}}{2 N}$. Then $h_{d y n}^{S Z}(\Theta, \mathcal{V}, \rho)=\ln 2$ and $h_{d y n}^{S Z}\left(\Theta^{2}, \mathcal{V}, \rho\right)=\frac{4}{3} \ln 2$.

Proof. Notice that from Equation (18), for each $m \in \mathbb{N}, n \in \mathbb{N}_{0}$ and $v_{0}, \ldots, v_{n} \in V$, we have

$$
\begin{aligned}
\mu^{\left(\Theta^{m}, \mathcal{V}, \rho\right)}\left(C\left(\begin{array}{ccc}
v_{0} & \cdots & v_{n} \\
0 & \cdots & n
\end{array}\right)\right) & :=\tau\left(\mathcal{V}\left(v_{n}\right) \circ \Theta^{m} \circ \cdots \circ \Theta^{m} \circ \mathcal{V}\left(v_{0}\right) \rho\right) \\
& =\operatorname{tr}\left(P_{v_{n}} U^{m} \cdots U^{m} P_{v_{0}} \rho P_{v_{0}}\left(U^{m}\right)^{*} \cdots\left(U^{m}\right)^{*} P_{v_{n}}\right) .
\end{aligned}
$$

Also, notice that $\rho=\frac{1}{2 N} \sum_{v \in V}(|R, v\rangle\langle R, v|+| L, v\rangle\langle L, v|)$ and so, for each $m \in \mathbb{N}$, Equation (36) becomes

$$
\begin{aligned}
\mu^{\left(\Theta^{m}, \mathcal{V}, \rho\right)}\left(C\left(\begin{array}{ccc}
v_{0} \cdots & \cdots & v_{n} \\
0 & \cdots & n
\end{array}\right)\right) & =\sum_{c \in\{R, L\}} \frac{1}{2 N} \operatorname{tr}\left(P_{v_{n}} U^{m} \cdots U^{m} P_{v_{0}}\left|c, v_{0}\right\rangle\left\langle c, v_{0}\right| P_{v_{0}}\left(U^{m}\right)^{*} \cdots\left(U^{m}\right)^{*} P_{v_{n}}\right) \\
& =\sum_{c, d \in\{R, L\}} \frac{1}{2 N}\left|\left\langle d, v_{n}\left|U^{m} P_{v_{n-1}} \cdots P_{v_{1}} U^{m}\right| c, v_{0}\right\rangle\right|^{2} .
\end{aligned}
$$

Let $\mathcal{A}$ be the atomic partition of $V$. We first show that $h_{\mathrm{dyn}}^{S Z}(\Theta, \mathcal{V}, \rho, \mathcal{A})=\ln 2$. Notice that for $(c, v) \in C \times V, U P_{v}|c, v\rangle=U|c, v\rangle$ and is given by Equation (32). Thus, by direct calculation, we have that

$$
\begin{aligned}
& \mu^{(\Theta, \mathcal{V}, \rho)}\left(C\left(\begin{array}{ccc}
v_{0} & \cdots & v_{n} \\
0 & \cdots & n
\end{array}\right)\right) \\
& =\sum_{c_{0}, c_{n} \in\{R, L\}} \frac{1}{2 N}\left|\left\langle c_{n}, v_{n}\left|U P_{v_{n-1}} \cdots P_{v_{1}} U\right| c_{0}, v_{0}\right\rangle\right|^{2} \text { by (37) } \\
& =\frac{1}{2 N} \sum_{c_{0}, c_{n} \in\{R, L\}} \mid\left.\left\langle c_{n}, v_{n}\right| U P_{v_{n-1}} \cdots P_{v_{1}}\left(\frac{1}{\sqrt{2}}\left(\left|R, v_{0}+1\right\rangle+(-1)^{\delta_{c_{0}, L}}\left|L, v_{0}-1\right\rangle\right)\right)\right|^{2} \\
& =\frac{1}{2 N} \sum_{c_{0}, c_{1}, c_{n} \in\{R, L\}} \frac{1}{2}\left(\delta_{v_{1}, v_{0}+1} \delta_{c_{1}, R}+\delta_{v_{1}, v_{0}-1} \delta_{c_{1}, L}\right)\left|\left\langle c_{n}, v_{n}\left|U P_{v_{n-1}} \cdots P_{v_{2}} U\right| c_{1}, v_{1}\right\rangle\right|^{2} \\
& =\cdots \\
& =\sum_{\substack{c_{k} \in\{R, L\} \\
0 \leq k \leq n}} \frac{1}{2 N} \prod_{k=1}^{n}\left(\frac{\delta_{v_{k}, v_{k-1}+1} \delta_{c_{k}, R}+\delta_{v_{k}, v_{k-1}-1} \delta_{c_{k}, L}}{2}\right) \\
& =\frac{1}{N} \prod_{k=1}^{n}\left(\frac{\delta_{v_{k}, v_{k-1}+1}+\delta_{v_{k}, v_{k-1}-1}}{2}\right) \text {, }
\end{aligned}
$$

for all $v_{0}, \ldots, v_{n} \in V$, which are exactly the probabilities $p_{\mathbf{X}}\left(v_{0}, \ldots, v_{n}\right)$ of a stationary Markov chain $\mathbf{X}$ which is governed by the transition matrix, $Q$, for the unbiased random walk on the $N$-cycle $V$. Therefore $h_{\mathrm{dyn}}^{S Z}(\Theta, \mathcal{V}, \rho, \mathcal{A})=H(Q)=\ln 2$, where the second equality follows from Proposition 2.20. Moreover, since $\widehat{\mathcal{A}}$ is a generating partition for $\left(V^{*}, \mathcal{P}(V)^{*}, \mu^{(\Theta, \mathcal{V}, \rho)}, s\right)$, we have that $h_{\mathrm{dyn}}^{S Z}(\Theta, \mathcal{V}, \rho)=\ln 2$ by Corollary 2.16,

Next we show that $h_{\text {dyn }}^{S Z}\left(\Theta^{2}, \mathcal{V}, \rho, \mathcal{A}\right)=\frac{4}{3} \ln 2$ using path counting techniques. To that end, for each $n \in \mathbb{N}_{0}$ and $(n+1)$-tuple $v=\left(v_{0}, \ldots, v_{n}\right) \in V^{n+1}$, we set

$$
l_{v}:=\mid\left\{k: k<n \text { such that } v_{k}=v_{k+1}=\cdots=v_{n}\right\} \mid .
$$


Then, we define the sets

$$
\begin{gathered}
L_{\mathrm{c}}^{n}:=\left\{\bar{v} \in V^{n+1}: \bar{v}=(v, \ldots, v) \text { for some } v \in V\right\}, \\
L_{\mathrm{e}}^{n}:=\left\{v \in V^{n+1}: l_{v} \text { is even }\right\} \backslash L_{\mathrm{c}}^{n} \text { and } L_{\mathrm{o}}^{n}:=\left\{v \in V^{n+1}: l_{v} \text { is odd }\right\} \backslash L_{\mathrm{c}}^{n} .
\end{gathered}
$$

For each $n \in \mathbb{N}_{0}$ and $v=\left(v_{0}, \ldots, v_{n}\right) \in V^{n+1}$, we will identify $v$ with the cylinder set $C\left(\begin{array}{ccc}v_{0} & \cdots & v_{n} \\ 0 & \cdots & n\end{array}\right)$ and consider $\mathcal{L}^{n}:=\left\{L_{\mathrm{c}}^{n}, L_{\mathrm{e}}^{n}, L_{\mathrm{o}}^{n}\right\}$ as a partition of $V^{*}$.

With this correspondence, we will show that the conditional probabilities, $p_{\mathbf{X}^{\left(\Theta^{2}, \mathcal{V}, \rho\right)}}\left(v_{n+1} \mid v\right)$, for $v_{n+1}$ given $v=\left(v_{0}, \ldots, v_{n}\right)$ are dependent upon which set $L \in \mathcal{L}^{n}$ that $v$ belongs to. First, we will determine the change of coin state that occurs after measuring the walker at the same site a number of times in a row. We claim that the resulting coin state, after $n$ measurements at a site $v$, depends only on the initial coin state $c \in\{R, L\}$ and the congruence class of $n$ modulo 4. Specifically, for all $n \in \mathbb{N}_{0}$, $\bar{v}=(v, \ldots, v) \in V^{n+1}$ and $c \in\{R, L\}$, we claim the following: if $n \equiv 0 \bmod 4$, then

$$
\underbrace{P_{v} U^{2} \cdots P_{v} U^{2}}_{n \text { times }}|c, v\rangle=a|c, v\rangle \text { for some } a \in \mathbb{C} \text { with }|a|=\frac{1}{2^{\left\lfloor\frac{n+1}{2}\right\rfloor}},
$$

if $n \equiv 1 \bmod 4$, then

$$
P_{v} U^{2} \cdots P_{v} U^{2}|c, v\rangle=a\left|L+(-1)^{\delta_{c, L}} R, v\right\rangle, \text { for some } a \in \mathbb{C} \text { with }|a|=\frac{1}{2^{\left\lfloor\frac{n+1}{2}\right\rfloor}},
$$

if $n \equiv 2 \bmod 4$, then

$$
P_{v} U^{2} \cdots P_{v} U^{2}|c, v\rangle=a\left|c^{\perp}, v\right\rangle \text { for some } a \in \mathbb{C} \text { with }|a|=\frac{1}{2^{\left\lfloor\frac{n+1}{2}\right\rfloor}},
$$

where we set $R^{\perp}=L$ and $L^{\perp}=R$, and, if $n \equiv 3 \bmod 4$, then

$$
P_{v} U^{2} \cdots P_{v} U^{2}|c, v\rangle=a\left|L-(-1)^{\delta_{c, L}} R, v\right\rangle \text {, for some } a \in \mathbb{C} \text { with }|a|=\frac{1}{2^{\left\lfloor\frac{n+1}{2}\right\rfloor}},
$$

where we used the abbreviation $|L \pm R, v\rangle:=|L, v\rangle \pm|R, v\rangle$. We will prove the claims by induction on $n$.

The base case, $n=0$, is trivial. For the inductive step we will handle the different congruence classes of $n$ separately. To this end, let $m \in \mathbb{N}$ with $m \geq 1$ and suppose that for all $n<m$ Equations (38)-(141) hold for all $\bar{v} \in V^{n+1}$ and their respective values of $n$. Fix $\bar{v} \in V^{m+1}$ and $c \in\{R, L\}$. If $m \equiv 1 \bmod 4$, then

$$
\begin{aligned}
P_{v} U^{2} \cdots P_{v} U^{2}|c, v\rangle & =P_{v} U^{2} a|c, v\rangle \text { for some } a \in \mathbb{C} \text { with }|a|=\frac{1}{2^{\left\lfloor\frac{m}{2}\right\rfloor}} \text { by (138) } \\
& =\frac{a}{2}\left|L+(-1)^{\delta_{c, L}} R, v\right\rangle \text { by (134) }
\end{aligned}
$$

and Equation (39) is satisfied since $\frac{1}{2 \cdot 2^{\left\lfloor\frac{m}{2}\right\rfloor}}=\frac{1}{2^{\left\lfloor\frac{m+1}{2}\right\rfloor}}$. If $m \equiv 2 \bmod 4$, then

$$
\begin{aligned}
P_{v} U^{2} \cdots P_{v} U^{2}|c, v\rangle & =P_{v} U^{2} a\left|L+(-1)^{\delta_{c, L}} R, v\right\rangle \text { for some } a \in \mathbb{C} \text { with }|a|=\frac{1}{2^{\left\lfloor\frac{m}{2}\right\rfloor}} \text { by (39) } \\
& =(-1)^{\delta_{c, L}} a\left|c^{\perp}, v\right\rangle,
\end{aligned}
$$

where the second equality holds because

$$
\begin{aligned}
& U^{2}|R+L, v\rangle=\sqrt{2} U|R, v+1\rangle=|L, v\rangle+|R, v+2\rangle \text { and } \\
& U^{2}|L-R, v\rangle=-\sqrt{2} U|L, v-1\rangle=|L, v-2\rangle-|R, v\rangle
\end{aligned}
$$


for all $v \in V$. Thus Equation (40) is satisfied since $\frac{1}{2^{\left\lfloor\frac{m}{2}\right\rfloor}}=\frac{1}{2^{\left\lfloor\frac{m+1}{2}\right\rfloor}}$. If $m \equiv 3 \bmod 4$, then

$$
\begin{aligned}
P_{v} U^{2} \cdots P_{v} U^{2}|c, v\rangle & =P_{v} U^{2} a\left|c^{\perp}, v\right\rangle \text { for some } a \in \mathbb{C} \text { with }|a|=\frac{1}{2^{\left\lfloor\frac{m}{2}\right\rfloor}} \text { by (40) } \\
& =\frac{a}{2}\left|L-(-1)^{\delta_{c, L}} R, v\right\rangle \text { by (34) }
\end{aligned}
$$

where we used the fact that $(-1)^{\delta_{c^{\perp}, L}}=-(-1)^{\delta_{c, L}}$ in the second equality. Hence Equation (41) is satisfied since $\frac{1}{2 \cdot 2^{\left\lfloor\frac{m n}{2}\right\rfloor}}=\frac{1}{2^{\left\lfloor\frac{m+1}{2}\right\rfloor}}$. If $m \equiv 0 \bmod 4$, then

$P_{v} U^{2} \cdots P_{v} U^{2}|c, v\rangle=P_{v} U^{2} a\left|L-(-1)^{\delta_{c, L}} R, v\right\rangle$ for some $a \in \mathbb{C}$ with $|a|=\frac{1}{2^{\left\lfloor\frac{m}{2}\right\rfloor}}$ by (41) $=-(-1)^{\delta_{c, L}} a|c, v\rangle$ by (42)

and hence Equation (38) is satisfied since $\frac{1}{2^{\left\lfloor\frac{m}{2}\right\rfloor}}=\frac{1}{2^{\left\lfloor\frac{m+1}{2}\right\rfloor}}$. Therefore the induction is complete and the claims are verified.

Next we claim that for all $v=\left(v_{0}, \ldots, v_{n}\right) \in V^{n+1} \backslash L_{\mathrm{c}}^{n}$ such that $p_{\mathbf{X}^{\left(\Theta^{2}, \mathcal{V}, \rho\right)}}(v) \neq 0$ there exists some $\psi \in H_{C}$ such that for all $v_{n+1} \in V$ the conditional pmf of $\mathbf{X}^{\left(\Theta^{2}, \mathcal{V}, \rho\right)}$ is given by

$$
p_{\mathbf{X}^{\left(\Theta^{2}, \mathcal{V}, \rho\right)}}\left(v_{n+1} \mid v_{0}, \ldots, v_{n}\right)=\frac{\sum_{d \in\{R, L\}}\left|\left\langle d, v_{n+1}\left|U^{2}\right| \psi, v_{n}\right\rangle\right|^{2}}{\|\psi\|^{2}} .
$$

Indeed, for all $v \in V^{n+1}$ with $p_{\mathbf{X}^{\left(\Theta^{2}, \mathcal{V}, \rho\right)}}(v) \neq 0$, we have

$$
\begin{aligned}
p_{\mathbf{X}^{\left(\Theta^{2}, \mathcal{V}, \rho\right)}}\left(v_{n+1} \mid v_{0}, \ldots, v_{n}\right) & =\frac{\mu^{\left(\Theta^{2}, \mathcal{V}, \rho\right)}\left(C\left(\begin{array}{ccc}
v_{0} & \cdots & v_{n+1} \\
0 & \cdots & n+1
\end{array}\right)\right)}{\mu^{\left(\Theta^{2}, \mathcal{V}, \rho\right)}\left(C\left(\begin{array}{ccc}
v_{0} & \cdots & v_{n} \\
0 & \cdots & n
\end{array}\right)\right)} \\
& =\frac{\sum_{c, d \in\{R, L\}}\left|\left\langle d, v_{n+1}\left|U^{2} P_{v_{n}} \cdots P_{v_{1}} U^{2}\right| c, v_{0}\right\rangle\right|^{2}}{\sum_{c, d \in\{R, L\}}\left|\left\langle d, v_{n}\left|U^{2} P_{v_{n-1}} \cdots P_{v_{1}} U^{2}\right| c, v_{0}\right\rangle\right|^{2}} \text { by (337) } \\
& =\sum_{c \in\{R, L\}} q_{c} \frac{\sum_{d \in\{R, L\}}\left|\left\langle d, v_{n+1}\left|U^{2} P_{v_{n}} \cdots P_{v_{1}} U^{2}\right| c, v_{0}\right\rangle\right|^{2}}{\sum_{d \in\{R, L\}}\left|\left\langle d, v_{n}\left|U^{2} P_{v_{n-1}} \cdots P_{v_{1}} U^{2}\right| c, v_{0}\right\rangle\right|^{2}},
\end{aligned}
$$

where, for each $c \in\{R, L\}$, we set

$$
q_{c}:=\frac{\sum_{d \in\{R, L\}}\left|\left\langle d, v_{n}\left|U^{2} P_{v_{n-1}} \cdots P_{v_{1}} U^{2}\right| c, v_{0}\right\rangle\right|^{2}}{\sum_{c^{\prime}, d \in\{R, L\}}\left|\left\langle d, v_{n}\left|U^{2} P_{v_{n-1}} \cdots P_{v_{1}} U^{2}\right| c^{\prime}, v_{0}\right\rangle\right|^{2}} .
$$

Notice that if $q_{c}=0$ in Equation (45) then the denominator on the right hand side of Equation (44) is also 0. In this case, we will use the convention that their product is defined and equal to 0 . For each $c \in\{R, L\}$, we define $\psi_{c} \in H_{C}$ to be the unique element satisfying the equation

$$
P_{v_{n}} U^{2} \cdots P_{v_{1}} U^{2}\left|c, v_{0}\right\rangle=\left|\psi_{c}, v_{n}\right\rangle .
$$

Then Equation (44) simplifies to

$$
p_{\mathbf{X}^{\left(\Theta^{2}, \mathcal{V}, \rho\right)}}\left(v_{n+1} \mid v_{0}, \ldots, v_{n}\right)=\sum_{c \in\{R, L\}} q_{c} \frac{\sum_{d \in\{R, L\}}\left|\left\langle d, v_{n+1}\left|U^{2}\right| \psi_{c}, v_{n}\right\rangle\right|^{2}}{\left\|\psi_{c}\right\|^{2}}
$$

where equality in the denominator follows by Parseval's identity. 
Notice that Equation (34) gives

$$
\begin{aligned}
& \operatorname{Ran}\left(P_{v+2} U^{2} P_{v}\right)=\operatorname{span}\{|R, v+2\rangle\} \text { and } \\
& \operatorname{Ran}\left(P_{v-2} U^{2} P_{v}\right)=\operatorname{span}\{|L, v-2\rangle\}, \text { for all } v \in V .
\end{aligned}
$$

Moreover, Equation (47) implies that, for any operator $A \in B(H)$,

$$
\begin{aligned}
& \operatorname{Ran}\left(P_{v+2} U^{2} P_{v} A\right) \subseteq \operatorname{span}\{|R, v+2\rangle\} \text { and } \\
& \operatorname{Ran}\left(P_{v-2} U^{2} P_{v} A\right) \subseteq \operatorname{span}\{|L, v-2\rangle\}, \text { for all } v \in V .
\end{aligned}
$$

Hence, if $v \in V^{n+1} \backslash L_{\mathrm{c}}^{n}$, then for each $c \in\{R, L\}$ we have

$$
P_{v_{n-l v}} U^{2} \cdots P_{v_{1}} U^{2}\left|c, v_{0}\right\rangle=a_{c}\left|d, v_{n-l_{v}}\right\rangle, \text { for some } a_{c} \in \mathbb{C},
$$

where $d=R$ whenever $v_{n-l_{v}}=v_{n-l_{v}-1}+2$ and $d=L$ when $v_{n-l_{v}}=v_{n-l_{v}-1}-2$. Thus $d$ does not depend on the initial coin state $c$. For each $d \in\{R, L\}$, we also have that

$$
P_{v_{n}} U^{2} \cdots P_{v_{n-l_{v}+1}} U^{2}\left|d, v_{n-l_{v}}\right\rangle=a\left|\psi, v_{n}\right\rangle \text {, for some } a \in \mathbb{C} \text { with }|a|=\frac{1}{2^{\left\lfloor\frac{l_{v}+1}{2}\right\rfloor}},
$$

where $\psi$ is the coin state given by Equations (38)-(41) depending on the congruence class of $l_{v}$ modulo 4 and we used the fact that $v_{n-l_{v}}=v_{n-l_{v}+1}=\cdots=v_{n}$ by definition of $l_{v}$. We combine Equations (48) and (49) to get that, for each $c \in\{R, L\}$,

$$
P_{v_{n}} U^{2} \cdots P_{v_{1}} U^{2}\left|c, v_{0}\right\rangle=a_{c}^{\prime}\left|\psi, v_{n}\right\rangle
$$

where $a_{c}^{\prime}=a_{c} \cdot a$ with $a_{c}$ and $a$ coming from Equations (48) and (49), respectively. Since $q_{R}+q_{L}=1$ and both $\psi_{R}$ and $\psi_{L}$ in Equation (46) are equal to $\psi$ which appears in Equation (50), we see that Equation (46) simplifies to Equation (43) as claimed.

Next we claim that, for $n \in \mathbb{N}_{0}$, if $v=\left(v_{0}, \ldots, v_{n}\right) \in L_{\mathrm{o}}^{n}$ and $p_{\mathbf{X}^{\left(\Theta^{2}, \mathcal{V}, \rho\right)}}(v) \neq 0$, then

$$
p_{\mathbf{X}^{\left(\Theta^{2}, \mathcal{V}, \rho\right)}}\left(v_{n+1} \mid v_{0}, \ldots, v_{n}\right)= \begin{cases}\frac{1}{2} & \text { if } v_{n+1}=v_{n} \\ \frac{1}{2} & \text { if } v_{n+1} \text { is exactly one of } v_{n} \pm 2 \\ 0 & \text { else }\end{cases}
$$

where the exactly one value of $v_{n+1} \in\left\{v_{n}-2, v_{n}+2\right\}$ with nonzero conditional probability depends on the given sequence $\left(v_{0}, \ldots, v_{n}\right)$ in the following manner:

- If

(i) $v_{n-l_{v}}=v_{n-l_{v}-1}+2$ and $l_{v}=1 \bmod 4$, or

(ii) $v_{n-l_{v}}=v_{n-l_{v}-1}-2$ and $l_{v}=3 \bmod 4$,

then $v_{n+1}=v_{n}+2$.

- If

(iii) $v_{n-l_{v}}=v_{n-l_{v}-1}-2$ and $l_{v}=1 \bmod 4$, or

(iv) $v_{n-l_{v}}=v_{n-l_{v}-1}+2$ and $l_{v}=3 \bmod 4$,

then $v_{n+1}=v_{n}-2$.

In addition we claim that, for $n \in \mathbb{N}_{0}$ and $v=\left(v_{0}, \ldots, v_{n}\right) \in V^{n+1}$, if $v \in L_{\mathrm{e}}^{n} \cup L_{\mathrm{c}}^{n}$ and $p_{\mathbf{X}^{\left(\Theta^{2}, \mathcal{V}, \rho\right)}}(v) \neq 0$, then

$$
p_{\mathbf{X}^{\left(\Theta^{2}, \mathcal{V}, \rho\right)}}\left(v_{n+1} \mid v_{0}, \ldots, v_{n}\right)= \begin{cases}\frac{1}{2} & \text { if } v_{n+1}=v_{n} \\ \frac{1}{4} & \text { if } v_{n+1}=v_{n}+2 \\ \frac{1}{4} & \text { if } v_{n+1}=v_{n}-2 \\ 0 & \text { else }\end{cases}
$$


In order to see Equation (51), let $v \in L_{\mathrm{o}}^{n}$ with $p_{\mathbf{X}^{\left(\Theta^{2}, \mathcal{V}, \rho\right)}}(v) \neq 0$ and suppose $v$ satisfies the conditions for Case (i); i.e. $v_{n-l_{v}}=v_{n-l_{v}-1}+2$ and $l_{v} \equiv 1 \bmod 4$. Since $v_{n-l_{v}}=v_{n-l_{v}-1}+2$, the coin state, $d$, on the right hand side of Equation (48) is $d=R$. Using this, the fact that $l_{v} \equiv 1 \bmod 4$ and Equation (39), we see that the coin state, $\psi$, on the right hand sides of Equations (49) and (50) is given by $\psi=R+L$. Plugging into Equation (43) and using (42), we have

$$
p_{\mathbf{X}^{\left(\Theta^{2}, \mathcal{V}, \rho\right)}}\left(v_{n+1} \mid v_{0}, \ldots, v_{n}\right)= \begin{cases}\frac{1}{2} & \text { if } v_{n+1}=v_{n} \\ \frac{1}{2} & \text { if } v_{n+1}=v_{n}+2 \\ 0 & \text { else }\end{cases}
$$

in this case. The other three cases can be done similarly and thus we obtain that Equation (51) is satisfied for all $v \in L_{\mathrm{o}}^{n}$.

Next, for the proof of Equation (52), let $v \in L_{\mathrm{e}}^{n}$ with $p_{\mathbf{X}^{\left(\Theta^{2}, \mathcal{V}, \rho\right)}}(v) \neq 0$. By Equations (38) and (40), we can see that the coin state $\psi$ in Equation (150) is given by $\psi=c$, for some $c \in\{R, L\}$. Plugging this value of $\psi$ into Equation (43) and using (35) we can see that the conditional pmf, $p_{\mathbf{X}^{\left(\Theta^{2}, \mathcal{V}, \rho\right)}}\left(v_{n+1} \mid v_{0}, \ldots, v_{n}\right)$, of $\mathbf{X}^{\left(\Theta^{2}, \mathcal{V}, \rho\right)}$ is given by Equation (52), for all $v \in L_{\mathrm{e}}^{n}$.

It remains only to show that Equation (52) is valid for all $\bar{v}=(v, \ldots, v) \in L_{\mathrm{c}}^{n}$. Since the modulus of $a$ in Equations (38)-(41) is independent of $c \in\{R, L\}$, we have $q_{c}=\frac{1}{2}$ in Equation (45) for both values of $c$. Note that by Equations (38)-(41) we have that the vector $\psi_{c} \in H_{C}$ which appears in Equation (46) is given by

$$
\psi_{c}= \begin{cases}c & \text { if } n \equiv 0 \bmod 4 \\ L+(-1)^{\delta_{c, L}} R & \text { if } n \equiv 1 \bmod 4 \\ c^{\perp} & \text { if } n \equiv 2 \bmod 4 \\ L-(-1)^{\delta_{c, L}} R & \text { if } n \equiv 3 \bmod 4\end{cases}
$$

Thus if $n$ is even then by Equations (46) and (35) we obtain immediately Equation (52). If $n$ is odd we examine the cases $n \equiv 1 \bmod 4$ and $n \equiv 3 \bmod 4$ separately. If $n \equiv 1 \bmod 4$ then $\psi_{R}=L+R, \psi_{L}=L-R$ and Equations (46) and (42) give Equation (52). The case of $n \equiv 3 \bmod 4$ can be verified similarly. that

We are now set to show $h_{\text {dyn }}^{S Z}\left(\Theta^{2}, \mathcal{V}, \rho, \mathcal{A}\right)=\frac{4}{3} \ln 2$. By direct calculation, we have

$$
\begin{aligned}
H_{\mu^{\left(\Theta^{2}, \mathcal{V}, \rho\right)}} & \left(X_{n+1}^{\left(\Theta^{2}, \mathcal{V}, \rho\right)} \mid\left(X_{0}^{\left(\Theta^{2}, \mathcal{V}, \rho\right)}, \ldots, X_{n}^{\left(\Theta^{2}, \mathcal{V}, \rho\right)}\right)\right) \\
& =\sum_{\substack{v_{k} \in V \\
0 \leq k \leq n}} p_{\mathbf{X}^{\left(\Theta^{2}, \mathcal{V}, \rho\right)}}\left(v_{0}, \ldots, v_{n}\right) \sum_{v_{n+1} \in V} \eta\left(p_{\mathbf{X}^{\left(\Theta^{2}, \mathcal{V}, \rho\right)}}\left(v_{n+1} \mid v_{0}, \ldots, v_{n}\right)\right) \text { by (8]) } \\
& =\mu^{\left(\Theta^{2}, \mathcal{V}, \rho\right)}\left(L_{\mathrm{e}}^{n} \cup L_{\mathrm{c}}^{n}\right)\left(2 \eta\left(\frac{1}{4}\right)+\eta\left(\frac{1}{2}\right)\right)+\mu^{\left(\Theta^{2}, \mathcal{V}, \rho\right)}\left(L_{\mathrm{o}}^{n}\right)\left(2 \eta\left(\frac{1}{2}\right)\right) \text { by ([51) and (52) } \\
& =\mu^{\left(\Theta^{2}, \mathcal{V}, \rho\right)}\left(L_{\mathrm{e}}^{n} \cup L_{\mathrm{c}}^{n}\right) \frac{3}{2} \ln 2+\mu^{\left(\Theta^{2}, \mathcal{V}, \rho\right)}\left(L_{\mathrm{o}}^{n}\right) \ln 2,
\end{aligned}
$$

where

$$
\mu^{\left(\Theta^{2}, \mathcal{V}, \rho\right)}\left(L_{x}^{n}\right)=\sum_{\left(v_{0}, \ldots, v_{n}\right) \in L_{x}^{n}} p_{\mathbf{X}^{\left(\Theta^{2}, \mathcal{V}, \rho\right)}}\left(v_{0}, \ldots, v_{n}\right) \text { for each } x \in\{\mathrm{c}, \mathrm{e}, \mathrm{o}\} .
$$


It remains only to solve for

$$
\lim _{n \rightarrow \infty} \mu^{\left(\Theta^{2}, \mathcal{V}, \rho\right)}\left(L_{x}^{n}\right) \text { for each } x \in\{\mathrm{c}, \mathrm{e}, \mathrm{o}\} .
$$

Notice that, by definition of $L_{\mathrm{c}}^{n}$,

$$
\mu^{\left(\Theta^{2}, \mathcal{V}, \rho\right)}\left(L_{\mathrm{c}}^{n}\right)=\sum_{v \in V} p_{\mathbf{X}^{\left(\Theta^{2}, \mathcal{V}, \rho\right)}}(\underbrace{v, \ldots, v}_{n+1 \text { times }}), \text { for each } n \in \mathbb{N}_{0} .
$$

Using Equation (52) $n$ times and Equation (37) to see that $p_{\mathbf{X}^{\left(\Theta^{2}, \mathcal{V}, \rho\right)}}(v)=\frac{1}{N}$, we obtain that $p_{\mathbf{X}^{\left(\Theta^{2}, \mathcal{V}, \rho\right)}}(\underbrace{v, \ldots, v}_{n+1 \text { times }})=\frac{1}{2^{n} N}$ and hence

$$
\mu^{\left(\Theta^{2}, \mathcal{V}, \rho\right)}\left(L_{\mathrm{c}}^{n}\right)=\frac{1}{2^{n}}, \text { for each } n \in \mathbb{N}_{0} .
$$

For ease of notation, set $e_{n}=\mu^{\left(\Theta^{2}, \mathcal{V}, \rho\right)}\left(L_{\mathrm{e}}^{n}\right), o_{n}=\mu^{\left(\Theta^{2}, \mathcal{V}, \rho\right)}\left(L_{\mathrm{o}}^{n}\right)$ and $c_{n}=\mu^{\left(\Theta^{2}, \mathcal{V}, \rho\right)}\left(L_{\mathrm{c}}^{n}\right)$, for each $n \in \mathbb{N}_{0}$. Notice that $e_{0}=o_{0}=0, c_{0}=1$ and $c_{n}=\frac{1}{2^{n}}$, for all $n \in \mathbb{N}$, by Equation (54). For each $v=\left(v_{0}, \ldots, v_{n-1}\right) \in V^{n}$ and $v_{n} \in V$ let $v \circ v_{n}=\left(v_{0}, \ldots, v_{n}\right) \in$ $V^{n+1}$. Suppose $v \in L_{\mathrm{o}}^{n-1}$. If $v_{n}=v_{n-1}$ then $l_{v}+1=l_{v \circ v_{n}}$ and if $v_{n} \neq v_{n-1}$ then $l_{v \circ v_{n}}=0$ and thus $p_{\mathbf{X}^{\left(\Theta^{2}, \mathcal{V}, \rho\right)}}\left(v \circ v_{n} \in L_{\mathrm{e}}^{n} \mid v \in L_{\mathrm{o}}^{n}\right)=1$ and $p_{\mathbf{X}^{\left(\Theta^{2}, \mathcal{V}, \rho\right)}}\left(v \circ v_{n} \in L_{x}^{n} \mid v \in\right.$ $\left.L_{\mathrm{o}}^{n}\right)=0$ for $x \in\{\mathrm{o}, \mathrm{c}\}$. Suppose $v \in L_{\mathrm{e}}^{n-1} \cup L_{\mathrm{c}}^{n-1}$. Then $v \circ v_{n} \in L_{\mathrm{e}}^{n}$ exactly when $v_{n} \neq v_{n-1}$. Therefore Equation (52) gives $p_{\mathbf{X}^{\left(\Theta^{2}, \mathcal{V}, \rho\right)}}\left(v \circ v_{n} \in L_{\mathrm{e}}^{n} \mid v \in L_{x}^{n}\right)=\frac{1}{2}$ for $x \in\{\mathrm{e}, \mathrm{c}\}$. Therefore

$$
e_{n}=o_{n-1}+\frac{1}{2}\left(e_{n-1}+c_{n-1}\right), \text { for all } n \in \mathbb{N} .
$$

Equation (52) also gives that $p_{\mathbf{X}^{\left(\Theta^{2}, \mathcal{V}, \rho\right)}}\left(v \circ v_{n} \in L_{\mathrm{o}}^{n} \mid v \in L_{\mathrm{e}}^{n}\right)=\frac{1}{2}$ and since $p_{\mathbf{X}^{\left(\Theta^{2}, \mathcal{V}, \rho\right)}}(v \circ$ $\left.v_{n} \in L_{\mathrm{o}}^{n} \mid v \in L_{\mathrm{c}}^{n}\right)=0$, we have

$$
o_{n}=\frac{1}{2} e_{n-1}, \text { for all } n \in \mathbb{N} .
$$

Therefore

$$
e_{n}=\frac{1}{2}\left(e_{n-1}+e_{n-2}+c_{n-1}\right), \text { for all } n \geq 2 .
$$

We claim that the $\operatorname{limits} e:=\lim _{n \rightarrow \infty} e_{n}$ and $o:=\lim _{n \rightarrow \infty} o_{n}$ both exist. It is enough, by Equation (56), to show that the limit $e$ exists. To see this we show that $\left(e_{n}\right)_{n \in \mathbb{N}_{0}}$ is increasing and bounded. We show that $\left(e_{n}\right)_{n \in \mathbb{N}_{0}}$ is increasing by induction. Since $e_{0}=0$ and $e_{1}=\frac{1}{2}$ (by Equation (155)), the base case is done. Next, fix $n \in \mathbb{N}$ with $n \geq 2$, suppose that $e_{m-1}<e_{m}$ for all $m \in\{1, \ldots, n-1\}$. Then, by Equation (57), it is enough to show that $e_{n-2}+c_{n-1}>e_{n-1}$. We see that,

$$
e_{n-1}=\frac{1}{2}\left(e_{n-2}+e_{n-3}+c_{n-2}\right)<e_{n-2}+c_{n-1},
$$

where the inequality follows by the inductive hypothesis and the fact that $\frac{1}{2} c_{n-2}=$ $c_{n-1}$. Therefore $\left(e_{n}\right)_{n \in \mathbb{N}_{0}}$ is increasing and trivially bounded by 1 and both the limits $e$ and $o$ exist. Furthermore, $1=e_{n}+o_{n}+c_{n}$, for all $n \in \mathbb{N}_{0}$, because $\mathcal{L}^{n}$ is a partition of $V^{*}$ and $\lim _{n \rightarrow \infty} c_{n}=0$ by Equation (54). Hence $1=e+o=\frac{3 e}{2}, e=\frac{2}{3}$ and $o=\frac{1}{3}$. Taking the limit in Equation (53), we see that

$$
\lim _{n \rightarrow \infty}\left(e_{n}+c_{n}\right) \frac{3}{2} \ln 2+o_{n} \ln 2=\frac{4}{3} \ln 2 .
$$


Therefore $h_{\mathrm{dyn}}^{S Z}\left(\Theta^{2}, \mathcal{V}, \rho\right)=h_{\mathrm{dyn}}^{S Z}\left(\Theta^{2}, \mathcal{V}, \rho, \mathcal{A}\right)=\frac{4}{3} \ln 2$ as desired.

The fact that $h_{\operatorname{dyn}}^{S Z}\left(\Theta^{2}, \mathcal{V}, \rho, \mathcal{A}\right) \neq h_{\operatorname{dyn}}^{S Z}\left(\Theta^{2}, \mathcal{T}, \rho, \mathcal{C}_{V}\right)$ provides further evidence of the sensitivity of quantum systems to measurement.

\section{REFERENCES}

1. L. Accardi, M. Ohya, and N. Watanabe, Dynamical entropy through quantum markov chains, Open Syst. Inf. Dyn. 4 (1997), no. 1, 71-87.

2. R. Alicki and M. Fannes, Defining quantum dynamical entropy, Lett. Math. Phys. 64 (1994), $75-82$.

3. A. Ambainis, E. Bach, A. Nayak, A. Vishwanath, and J. Watrous, One-dimensional quantum walks, Proc. of the Thirty-third Annual ACM Symposium on Theory of Computing (New York), STOC '01, ACM, 2001, pp. 37-49.

4. L. Boltzmann, Über die mechanische bedeutung des zweiten hauptsatzes der wrmetheorie, Wiener Berichte 53 (1866), 195-220.

5. A. Connes, H. Narnhofer, and W. Thirring, Dynamical entropy of $C^{*}$ algebras and von Neumann algebras, Comm. Math. Phys. 112 (1987), 691-719.

6. T. M. Cover and J. A. Thomas, Elements of information theory, Wiley Interscience, New York, 1991.

7. E. B. Davies, Quantum theory of open systems, Academic Press Inc., London, Nov 1976.

8. E. B. Davies and J. T. Lewis, An operational approach to quantum probability, Comm. Math. Phys. 17 (1970), no. 3, 239-260.

9. T. Downarowicz, Entropy in dynamical systems, Cambridge University Press, New York, 2011.

10. C. M. Edwards, The algebraic approach to algebraic quantum theory I, Comm. Math. Phys. 16 (1970), no. 3, 207-230.

11. T. Endo and N. Konno, The stationary measure of a space-inhomogeneous quantum walk on the line, arXiv preprint arXiv:1309.3054 (2014).

12. J. Kempe, Quantum random walks an introductory overview, Contemporary Phys. 44 (2003), no. 4, 307-327.

13. B. P. Kitchens, Symbolic dynamics: One-sided, two-sided, and countable state Markov shifts, Springer, 1998.

14. N. Konno, T. Luczak, and E. Segawa, Limit measures of inhomogeneous discrete-time quantum walks in one dimension, Quantum Inf. Process. 12 (2013), 33-53.

15. A. Kossakowski, M. Ohya, and N. Watanabe, Quantum dynamical entropy for completely positive maps, Infin. Dimens. Anal. Quantum Probab. Relat. Top. 2 (1999), no. 2, 267-282.

16. N. B. Lovett, S. Cooper, M. Everitt, M. Trevers, and V. Kendon, Universal quantum computation using the discrete-time quantum walk, Phys. Rev. A 81 (2010), no. 4, 042330.

17. R. Portugal, Quantum random walks and search algorithms, Springer, New York, 2013.

18. C. E. Shannon, A mathematical theory of communication, Bell System Tech. J. 27 (1948), no. 3, 379-423.

19. C. E. Shannon, A mathematical theory of communication, Bell System Tech. J. 27 (1948), no. 4, 623-666.

20. W. Słomczyński and A. Szczepanek, Quantum dynamical entropy, chaotic unitaries and complex Hadamard matrices, IEEE Trans. Inform. Theory 63 (2017), no. 12, 7821-7831.

21. W. Słomczyński and K. Życzkowski, Quantum chaos: An entropy approach, J. Math. Phys. 35 (1994), no. 2, 5674-5700.

22. P. Walters, An introduction to ergodic theory, Springer-Verlag, New York, 1982.

Department of Mathematics, University of South Carolina, Columbia, SC 29208 U.S.A.

E-mail address: giorgis@math.sc.edu

E-mail address: dw7@math.sc.edu 